\title{
Shear Band Localization Via Local J2 Continuum Damage Mechanics
}

\author{
M. Cervera \\ M. Chiumenti \\ C. Agelet de Saracibar
}




\section{Shear Band Localization Via Local J2 Continuum Damage Mechanics}

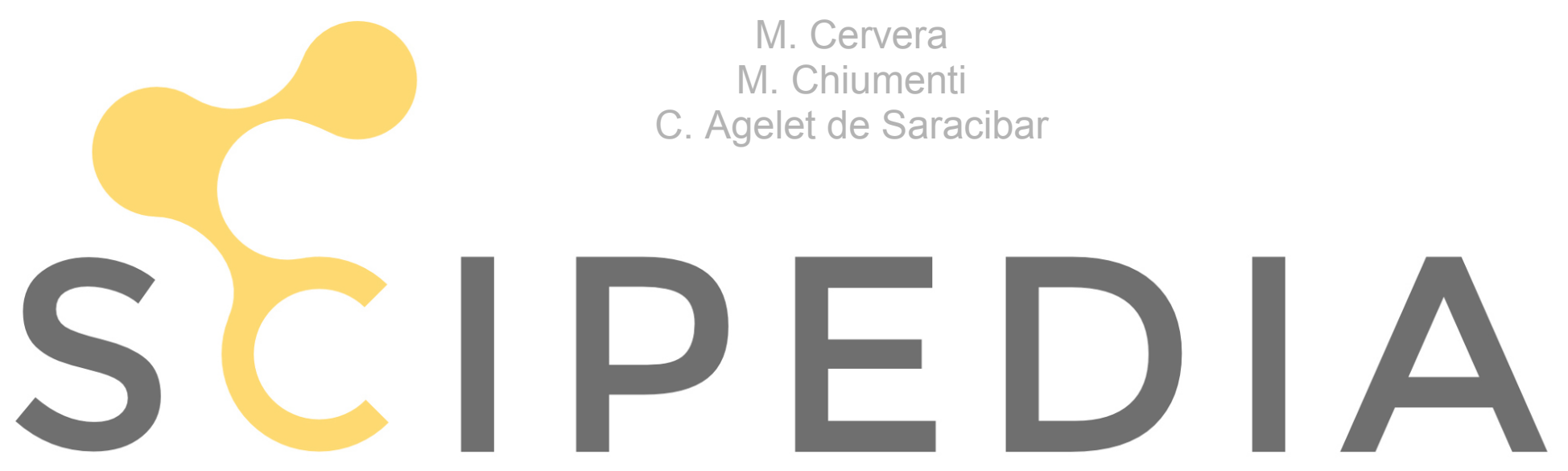

Register for free at https//www.scipedia.com to download the version without the watermark

Monograph CIMNE Nº-78, July 2003 


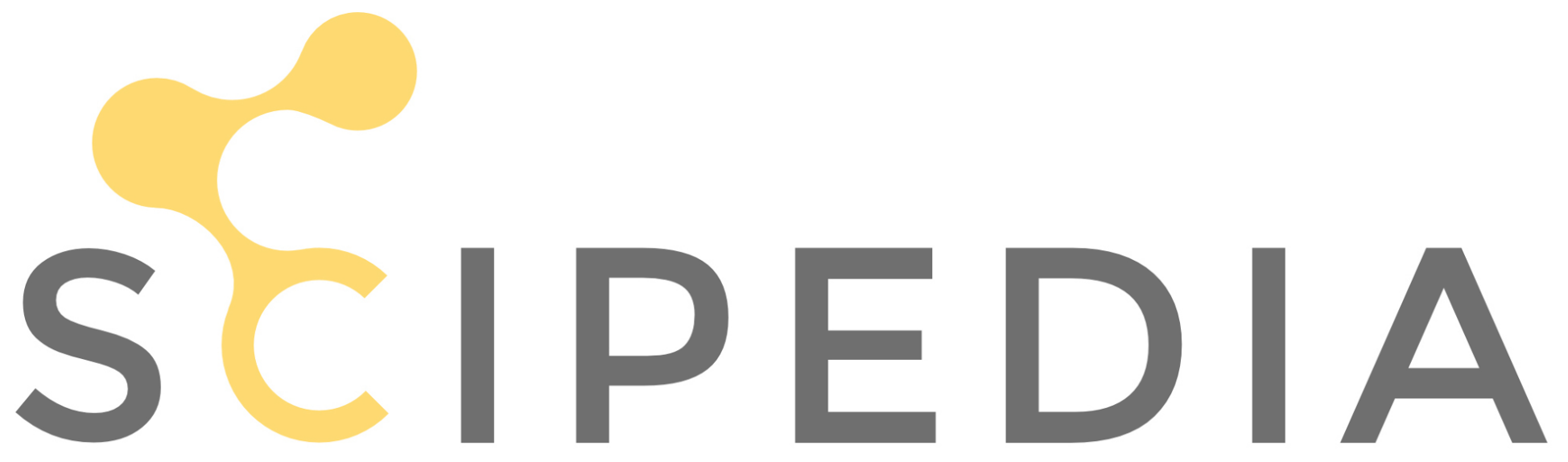

Register for free at https//www.scipedia.com to download the version without the watermark

INTERNACIONAL CENTER FOR NUMERICAL METHODS IN ENGINEERING

Edificio C1, Campus Norte UPC

Gran Capitán s/n

08034 Barcelona, Spain

www.cimne.upc.es

First edition: July 2003

SHEAR BAND LOCALIZATION VIA LOCAL J2 CONTINUUM DAMAGE MECHANICS

Monograph CIMNE M78

(c) The authors

ISBN: 84-95999-36-6

Depósito legal: B-37429-2003 


\section{Shear band localization via local $\mathrm{J}_{2}$ continuum}

damage mechanics
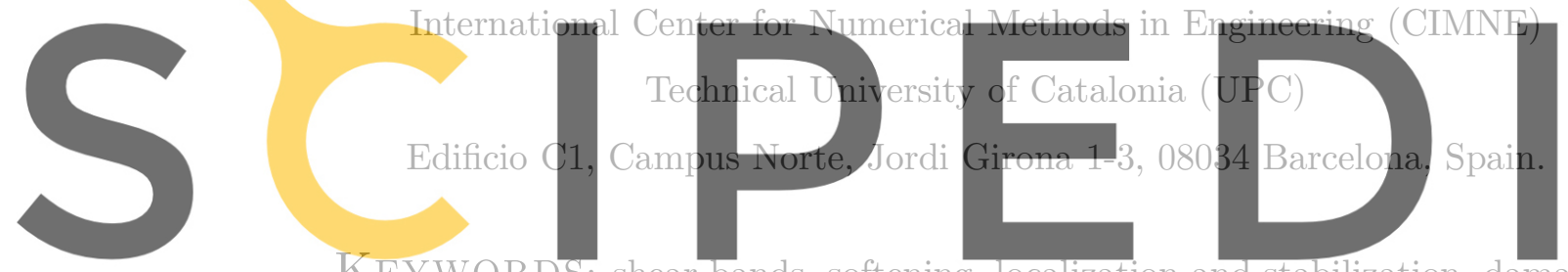

M. Cervera, M. Chiumenti and C. Agelet de Saracibar

KEYWORDS: shear bands, softening, localization and stabilization, damage,

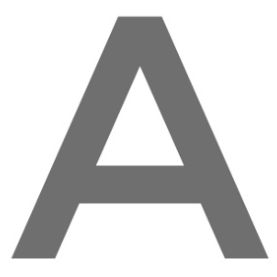

Register for free at https//www.scipedia.copqntorewilithoad the version without the watermark 


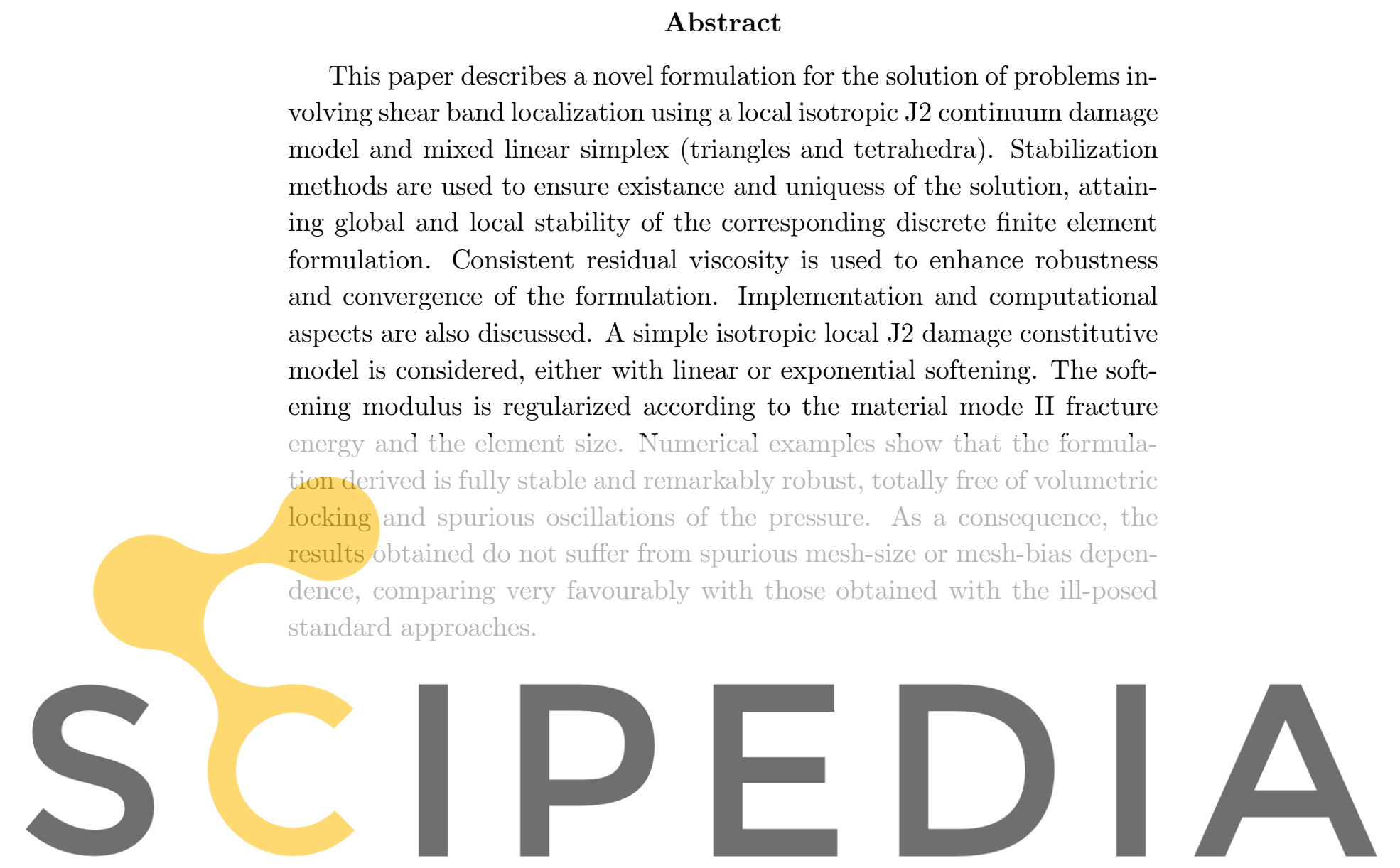




\section{Index}

$\begin{array}{llr}1 & \text { Introduction } & 7\end{array}$

2 Boundary value problem 13

2.1 Stress and strain tensors . . . . . . . . . . . . . . 13

2.2 Strong and weak forms . . . . . . . . . . . . . . . . . . . 14

2.3 Irreductible, mixed and stabilized methods . . . . . . . . . . . . 14

2.4 The Sub-Grid Scale approach . . . . . . . . . . . 17

2.5 Orthogonal Sub-Grid Scales (OSGS) and Galerking Least Squares
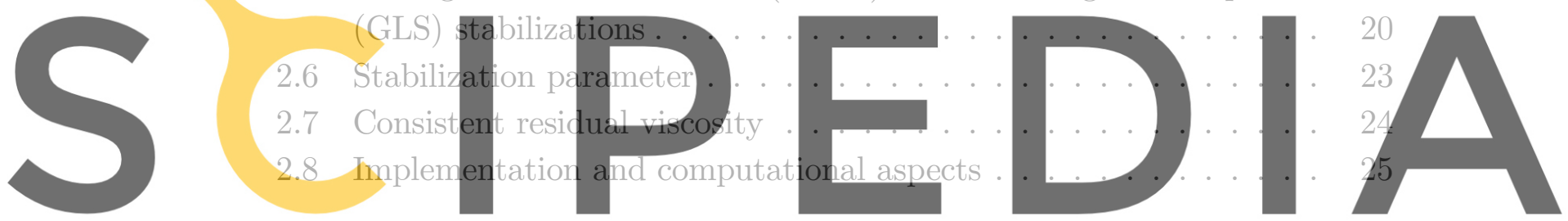

3 Mixed formulation for J2 damage

29

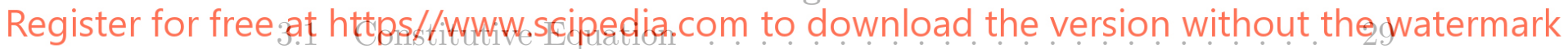

3.2 Characterization of Damage . . . . . . . . . . . . 29

3.3 Rate dependent damage . . . . . . . . . . . . . . . . . 32

3.4 Thermodynamic Framework . . . . . . . . . . . . . . . . 33

3.5 Softening behaviour and regularization . . . . . . . . . . . . 34

3.5.1 Rate independent behaviour . . . . . . . . . . . . . . 35

3.5.2 Rate dependent behaviour . . . . . . . . . . . . 36

3.6 Update of the damage threshold . . . . . . . . . . . . . . 37

3.7 Tangent operator . . . . . . . . . . . . . . . . . . . 38

3.7 .1 Rate independent damage . . . . . . . . . . . . . . . . 38

3.7.2 Rate dependent damage . . . . . . . . . . . . . . . 40

3.8 Stabilization parameter . . . . . . . . . . . . . . . 40 
4 Numerical examples $\quad 41$

4.1 Singly perforated strip . . . . . . . . . . . . . . . . 42

4.2 Doubly perforated strip . . . . . . . . . . . . . . . . . . . . . . . . . . . . . 48

4.3 Multiply perforated strip . . . . . . . . . . . . . . . 55

5 Conclusions

63
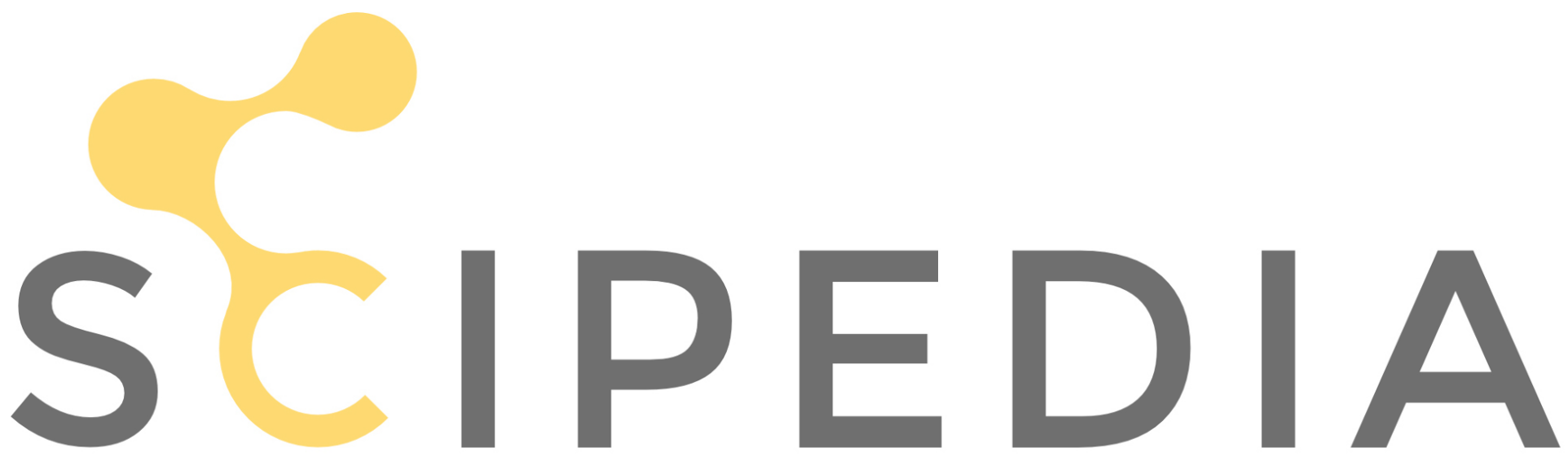

Register for free at https//www.scipedia.com to download the version without the watermark 


\section{List of Figures}

1.1 Strain localization: (a) weak discontinuity; (b) strong discontinuity 8

2.1 Continuum and discrete compressible irreductible elliptic problem 16

2.2 Continuum and discrete incompressible mixed non-elliptic problem 16

3.1 J2-damage model: (a) damage surface, (b) softening functions . . 30

4.1 Original and deformed (x 5) geometries for singly perforated strip 42

4.2 Results for singly perforated strip using the standard irreductible formulation. Contours for: (a) vertical displacement, (b) equiva-

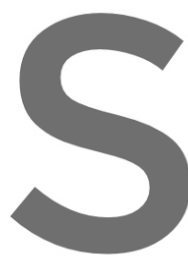
lent deviatoric

\section{3} Results for sing tion. Contours atoric strain, (c) damage index a
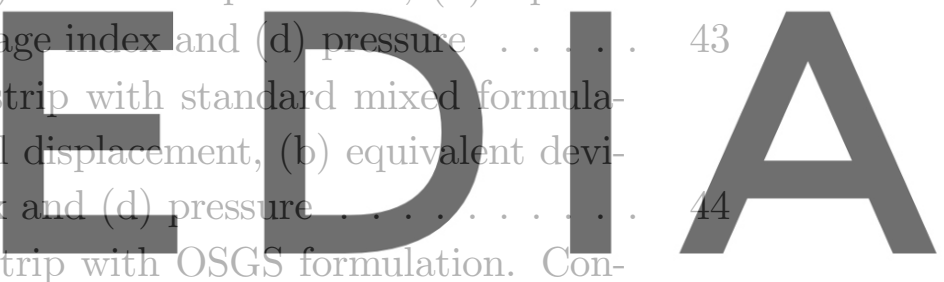

Register for free at https / Jwww.scipedia.com to download the version without the watermark (c) damage index and (d) pressure..... . . . . . . . 45

4.5 Evolution of damage for singly perforated strip. Contours for (half)-imposed vertical displacement equal to: (a) 0.010, (b) 0.015, (c) 0.020 and $(\mathrm{d}) 0.025 \ldots \ldots \ldots \ldots$

4.6 Load versus displacement for simply perforated strip. Comparison among different formulations . . . . . . . . . . . . . . 47

4.7 Load versus displacement for simply perforated strip. Comparison between different mesh sizes . . . . . . . . . . . . . . . 47

4.8 Original and deformed (x 5) geometries for doubly perforated strip 49

4.9 Results for doubly perforated strip using the standard irreductible formulation. Contours for: (a) vertical displacement, (b) equivalent deviatoric strain, (c) damage index and (d) pressure . . . . 50 
4.10 Results for doubly perforated strip with standard mixed formulation. Contours for: (a) vertical displacement, (b) equivalent deviatoric strain, (c) damage index and (d) pressure . . . . . . . . 51

4.11 Results for doubly perforated strip with OSGS formulation. Contours for: (a) vertical displacement, (b) equivalent deviatoric strain, (c) damage index and (d) pressure . . . . . . . . . . . . 52

4.12 Evolution of damage for doubly perforated strip. Contours for (half)-imposed vertical displacement equal to: (a) 0.010, (b) 0.020,

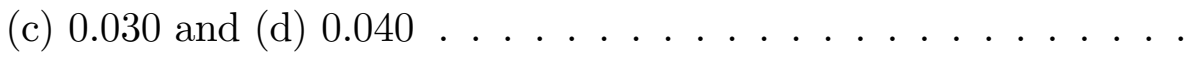

4.13 Load versus displacement for doubly perforated strip. Comparison among different formulations . . . . . . . . . . . . 54

4.14 Load versus displacement for doubly perforated strip. Comparison between different mesh sizes

4.15 Original and deformed ( $\mathrm{x}$ 5) geometries for multiply perforated strip

4.16 Results for multiply perforated strip using the standard irreductible formulation. Contours for: (a) vertical displacement, (b) equiva-
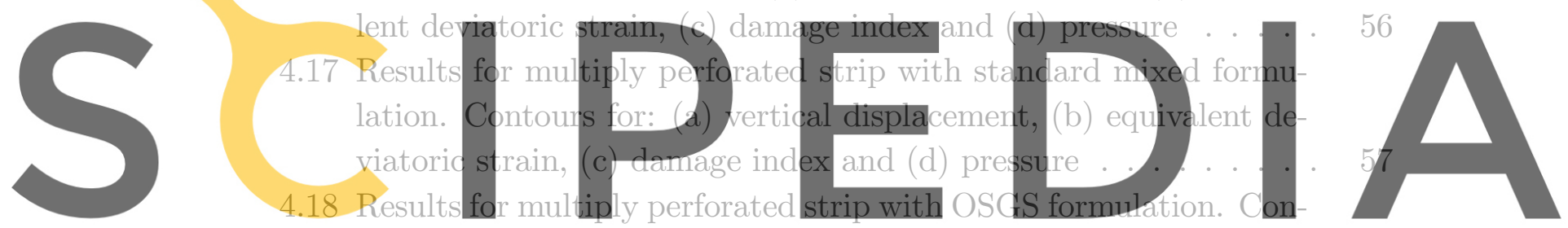

tours for: (a) vertical displacement, (b) equivalent deviatoric strain,

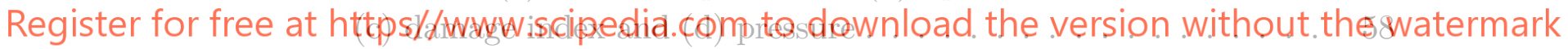

4.19 Evolution of damage for multiply perforated strip. Contours for (half)-imposed vertical displacement equal to: (a) 0.010, (b) 0.015, (c) 0.020 and $(\mathrm{d}) 0.025 \ldots \ldots \ldots$. . . . . . . . . . . . . . . 59

4.20 Load versus displacement for multiply perforated strip. Comparison among different formulations . . . . . . . . . . .

4.21 Load versus displacement for multiply perforated strip. Comparison between different mesh sizes . . . . . . . . . . . . . . . 


\section{Introduction}

It is well established that softening materials subjected to monotonic straining exhibit strain localization. In particular, in the so-called J2 materials, shear (or slip) strains concentrate, under certain circumstances. This phenomenon leads to the formation of shear bands inside the solid where, once the peak stress is reached, the deformation concentrates while the material outside the band unloads. This induces the formation of weak discontinuities, with continuous fields of slip displacements but discontinuous fields of shear strain inside and
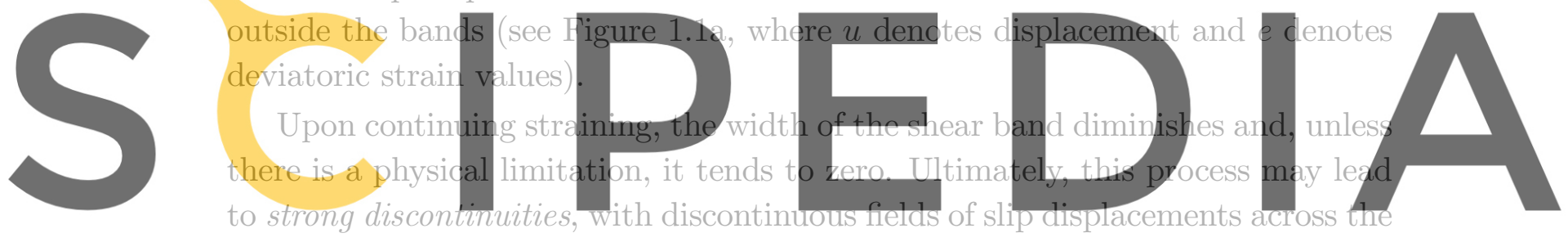

Regin discontinuity line, and unbounded shear strains (see Figure 1.11). In 32 materials, Register for free at https//www.scipedia.com to download the version without the watermark these are called slip lines. It is generally accepted in macture mechanics that the amount of energy released during the formation of a unit area of slip surface is a material property, called the mode II fracture energy.

Shear bands are typical of ductile materials such as metals, although they are also observed in granular materials such as sands or soils. Similar deformation patterns can also appear in fiber-reinforced composites subjected to compressive loading.

In the last two decades, many different finite element strategies have been proposed to model discontinuities and the references in the bibliography are innumerable. Despite the considerable effort devoted to the subject, theoretical modeling and computational resolution of the strain localization process up to structural failure have remained an open challenge in Computational Solid Me- 

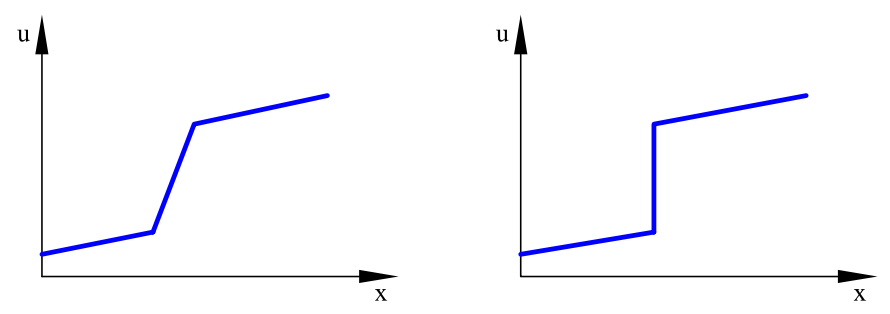

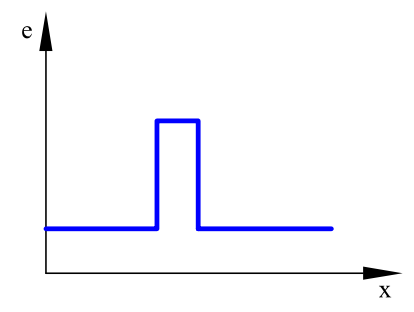

a)

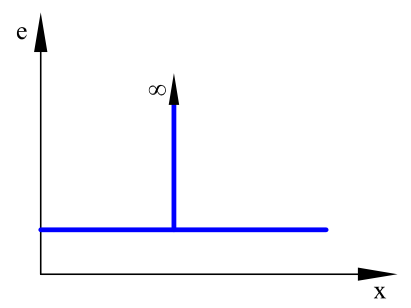

b)

Fig. 1.1: Strain localization: (a) weak discontinuity; (b) strong discontinuity

chanics.

The possibilities to model shear bands with finite elements are several, and both the weak and the strong discontinuity approaches have been followed. In the first, the objective is to capture the shear band as precisely as possible, with standard continuous elements. In the second, the displacement field is enhanced with discontinuous functions so that the true slip line can be captured. In fact, both approaches are compatible. On one hand, a weak discontinuity can be interpreted as a regularization of a strong one over a given width, for instance with the discontinuity "smeared" across the maximum possible resolution of the mesh, that is, one element; on the other hand, a strong discontinuity is the limit case of a weak one with vanishing width.

The main difficulty why most attempts to model weak displacement discontinuities with standard, local, approaches is that the solutions obtained appear to be unphysically, and unacceptably, fully determined by the fineness and orientation of the discretization. Up to now, this disagreeable observation has been erroneously and misleadingly attributed to the fact that, when strain-softening occurs and the slope of the local stress-strain curve becomes negative, the governing equations of the Continuum Mechanics problem lose their "natural" elliptic 
character. To remedy this, many so-called "non-local", "gradient-enhanced", "micropolar" and "viscous-regularized" constitutive models have been proposed in the last decade.

In the first, non-local, strategy, the standard format of the constitutive relationships (stress at a point depends on the strain at that point) is substituted by a non-local format (stress at a point depends on some average measure of the strain in the neighbourhood of that point), see [1], [2], among others. In the second, gradient-enhanced, strategy, a variant of the first, the nonlinear constitutive laws, for plasticity or damage, are made dependent not only on the local inelastic strain, but also on its second gradient, which is computed according to some additional relation which couples it to the local strain, [3], [4], [5], [6], [7], [8], [1]. In the third, micropolar, strategy, the usual non-polar description of Continuum Mechanics is substituted by other non-standard theory, like the Cosserat's continuum, see, for instance, [9] and [10]. In the fourth, viscous-regularized, strategy, the rate-independent format is substituted by a regularized version, also dependent on the strain-rate, see [11], [8], [1].

Even if these strategies have proved effective to some extend, they also pose new theoretical and computational difficulties, not fully mastered at the present moment.

Alternatively, in the last decade, an effort has been made to model numerically strong discontinuities directly ([12], [13], [14]). Again, even if the strong discontinuity concept is certainly appealing, it departs from the usual Continuum Mechanics framework and requires a new solid theoretical basis. This leads to several new formulations for finite elements with "embedded discontinuities", depending on the kinematical and statical assumptions adopted, all of them still with open questions. For instance, the issue of uniqueness of the solution is not fully solved yet. They also pose new computational challenges, as their application invariably needs the use of "tracking" algorithms, in order to properly follow the progress of the strong discontinuity. It is not easy for these tracking algorithms to keep up with the progress of multiple, interconnected or branching discontinuities [15]. Also, applications in 3D are still a real challenge, although some progress has been made in the last years [16].

It will be shown in this paper that the difficulties encountered in localization problems are not related to the local definition of the constitutive laws.

Surprisingly, up to now, most of the studies about shear localization have 
been conducted using J2 plastic or isotropic damage models, implemented within the standard irreducible finite element formulation, with the displacement field as only unknown variable. Unfortunately, J2 inelastic deformation is isochoric, and the irreducible formulation is not well suited to cope with the corresponding incompressibility constraint. The unsuitability of the irreductible formulation is more evident if low order finite elements are used and, very especially, for simplicial elements (triangles and tetrahedra).

However, the mixed displacement/pressure $(\mathbf{u} / p)$ formulation is the appropriate framework to tackle (quasi)-incompressible problems [18]. In fact, very promising results have been obtained in localization problems with J2 plasticity using this formulation together with remeshing techniques in [19] and [20]. Nevertheless, it is difficult to construct stable low order elements and, again, particularly, low order simplex. This is another very attractive area of on-going research, see references [21], [22], [23], [24], [25], [26], [27], [28], [29], [30], among others.

Recently, the authors have applied stabilization methods, originated in the field of Computational Fluid Dynamics ([31], [32], and others), to the solution of incompressible elasto-plastic problems with mixed displacement/pressure linear/linear simplicial elements, see [33], [34] and [35]. In reference [36] it was demonstrated that stable methods can be developed for softening J2-plastic materials, while retaining the local character of the constitutive behaviour. This translates in the achievement of three important goals: (a) the solution of the corresponding localization boundary value problem exists and it is unique, (b) the position and orientation of the localization bands is independent of the directional bias of the finite element mesh and (c) the global post-peak load-deflection curves are independent of the size of the elements in the localization band. The accomplishment of these fundamental objectives is attained by ensuring both global and local stability of the problem; as it will be shown, this is secured by the appropriate modification of the variational formulation, making use of the concept of sub-grid approximation.

Shear bands are not exclusive of J2-plastic materials. In the present work we show that an isotropic continuum J2-damage model can equally lead to shear localization. Since the Continuum Damage Theory was firstly introduced by Kachanov [37] 45 years ago, it has been widely accepted as an alternative to deal with complex material behaviour. The published references are innumerable, 
as the theory has been used to model creep damage, fatigue damage, elastodamage, ductile plastic damage, as well as the behaviour of brittle materials. The reason for its popularity is as much the intrinsic simplicity and versatility of the approach, as well as its consistency, based on the theory of thermodynamics of irreversible processes. Reference [17] presents an up-to-date review of damagebased approaches for the fracture of quasi-brittle materials, linking them to the now old-fashioned, although still very popular, "smeared crack" models of the 1980's.

In this work, we formulate a local isotropic J2-damage model, with only one scalar internal variable to monitor the local deviatoric damage. This choice implies that the macroscopic anisotropy of the structural behaviour has to be captured by means of a finite element approximation to within the resolution of the adopted mesh [44], [45], [46]. This provides an extremely simple constitutive model which, nevertheless, is able to predict correctly the softening response. Furthermore, the model can be implemented in a strain-driven form which leads to a closed-form algorithm to integrate the stress tensor. This is a most valuable feature for a model intended to be used in large scale 2D and 3D computations.

The outline of this work is as follows. In the first chapter, the corresponding mixed displacement/pressure $(\mathbf{u} / p)$ boundary value problem is formulated. Strong and weak forms are presented and the difficulties of solving localization problems using the standard, irreductible or mixed, Galerkin formulations are explained. It is shown how the mixed problem can be globally and locally stabilized with the Orthogonal SubGrid Scale (OSGS) and Galerkin Least Square (GLS) methods. The possibility of enhancing the robustness and convergence of the numerical procedure by the inclusion of consistent residual viscosity is also considered. Implementation and computational aspects are discussed next. In the second chapter, the mixed isotropic continuum J2-damage model is presented. The necessary regularization of the softening modulus according to the size of the elements inside the localization band is discussed. Finally, in the third chapter, three numerical examples are presented to assess the present formulation and to compare its performance with those of the standard irreductible and mixed Galerkin triangular and tetrahedral elements. 


\section{Boundary value problem}

\subsection{Stress and strain tensors}

The natural formulation of the incompressible mechanical problem considers the hydrostatic pressure $p$ as an independent unknown, additional to the primary displacement field. The (second order) stress tensor $\boldsymbol{\sigma}$ is then expressed as:

$$
\boldsymbol{\sigma}=p \mathbf{1}+\mathbf{s}
$$

where $p=\frac{1}{3} \operatorname{tr}(\boldsymbol{\sigma})$ and $\mathbf{s}=\operatorname{dev}(\boldsymbol{\sigma})$ are the volumetric and the deviatoric parts of the stress tensor, respectively, and $\mathbf{1}$ is the (second order) unit tensor. Correspondingly, the (second order) strain tensor $\varepsilon=\nabla^{s} \mathbf{u}$, where $\mathbf{u}$ are the displacements, is expressed as:

$$
\boldsymbol{\varepsilon}(\mathbf{u})=\frac{1}{3} \varepsilon_{v} \mathbf{1}+\mathbf{e}
$$

where $\varepsilon_{v}=\operatorname{tr} \varepsilon=\nabla \cdot \mathbf{u}$ and $\mathbf{e}=\operatorname{dev} \varepsilon$ are the volumetric and the deviatoric parts of the strain tensor, respectively. For linear elastic behaviour, the constitutive equations are expressed as:

$$
\begin{aligned}
p & =K \varepsilon_{v} \\
\mathbf{s} & =2 G \operatorname{dev} \boldsymbol{\varepsilon}=2 G \mathbf{e}
\end{aligned}
$$

where $K$ is the bulk modulus, also referred to as modulus of volumetric compressibility, and $G$ is the shear modulus. In incompressible elasticity, $K$ tends to infinity and, thus, $\varepsilon_{v}=\nabla \cdot \mathbf{u}=0$. 


\subsection{Strong and weak forms}

The strong form of the continuum mechanical problem can be stated as: find the displacement field $\mathbf{u}$ and the pressure field $p$, for given prescribed body forces $\mathbf{f}$, such that:

$$
\begin{aligned}
\nabla \cdot \mathbf{s}+\nabla p+\mathbf{f} & =\mathbf{0} & & \text { in } \Omega \\
\nabla \cdot \mathbf{u}-\frac{1}{K} p & =0 & & \text { in } \Omega
\end{aligned}
$$

where $\Omega$ is the open and bounded domain of $\mathbb{R}^{n_{\text {dim }}}$ occupied by the solid in a space of $n_{\text {dim }}$ dimensions. Eqs. (2.5)-(2.6) are subjected to appropriate Diritchlet and Neumann boundary conditions. In the following, we will assume these in the form of prescribed displacements $\mathbf{u}=\overline{\mathbf{u}}$ on $\partial \Omega_{u}$, and prescribed tractions $\overline{\mathbf{t}}$ on $\partial \Omega_{t}$, respectively. In the mixed formulation the value of the pressure is defined by the Neumann conditions or, alternatively, by prescribing its value at some point.

The associated weak form of the problem $(2.5)+(2.6)$ can be stated as:

$$
\begin{aligned}
(\mathbf{v}, \nabla \cdot \mathbf{s})+(\mathbf{v}, \nabla p)+(\mathbf{v}, \mathbf{f}) & = & 0 & \forall \mathbf{v} \\
(q, \nabla \cdot \mathbf{u})-\left(q, \frac{1}{K} p\right) & =0 & & \forall q
\end{aligned}
$$

where $\mathbf{v} \in \mathcal{V}$ and $q \in \mathcal{Q}$ are the variations of the displacements and pressure fields, respectively, $\mathcal{V}=H_{0}^{1}(\Omega)$ is the space of continuous functions with discontinuous derivatives, $\mathcal{Q}=L^{2}(\Omega)$ is the space of square integrable functions in $\Omega$ and $(\cdot, \cdot)$ denotes the inner product in $L^{2}(\Omega)$. Integrating Eq. (2.7) by parts, the problem can be rewritten in the standard form as:

$$
\begin{aligned}
\left(\nabla^{s} \mathbf{v}, \mathbf{s}\right)+(\nabla \cdot \mathbf{v}, p)-(\mathbf{v}, \mathbf{f})-(\mathbf{v}, \overline{\mathbf{t}})_{\partial \Omega} & =0 & \forall \mathbf{v} \\
(q, \nabla \cdot \mathbf{u})-\left(q, \frac{1}{K} p\right) & =0 & \forall q
\end{aligned}
$$

\subsection{Irreductible, mixed and stabilized methods}

Over the last years, many researchers have supported the misleading idea that the underlying reason why the standard, local, rate-independent constitutive models are inadequate to model zones of localized straining correctly is the local change 
of character of the governing equations (see [1], [2], [3], [4], [5], [6], [7], [8], [9], [7], [10] and many others.).

In fact, substituting Eq. (2.6) into Eq. (2.5), and reducing the discussion to standard elasticity, yields the irreductible form:

$$
G \Delta \mathbf{u}+K \nabla(\nabla \cdot \mathbf{u})+\mathbf{f}=\mathbf{0} \quad \text { in } \Omega
$$

where $\Delta(\cdot)$ denotes the laplacian operator and $G$ and $K$ are the shear and bulk modulus, respectively.

A standard stability (or energy) estimate for problem (2.11) is obtained by multiplying the left hand side by $\mathbf{u}$ and integrating by parts over the domain $\Omega$, to yield

$$
\begin{aligned}
G(\nabla \mathbf{u}, \nabla \mathbf{u})+K(\nabla \cdot \mathbf{u}, \nabla \cdot \mathbf{u}) & =G\|\nabla \mathbf{u}\|^{2}+K\|\nabla \cdot \mathbf{u}\|^{2} \\
& =\|\mathbf{u}\|_{E}^{2}
\end{aligned}
$$

where $\|\cdot\|_{E}^{2}$ is the energy norm (equal to the elastic free energy). From Eqs. (2.12)(2.13), the elliptic character of the original equations is evident, for $G, K \geq 0$. It is, therefore, obvious that in nonlinear solid continuum mechanics with softening, where the local tangent values of the moduli become negative, the rate equations lose ellipticity. It is important to note that as long as the secant moduli remain positive, the equations in terms of the total displacement $\mathbf{u}$ (non its increments) remain elliptic.

Anyway, loss of ellipticity does not mean that the problem be ill-posed or that it can not be solved numerically. Parabolic and hyperbolic partial differential equations certainly have solutions and they can be computed, analytically or numerically.

The true panorama is as follows. For a compressible irreductible elliptic problem in the continuum, it can be proved that the solution $\mathbf{u} \in \mathcal{V}$ exists and it is unique, $\mathcal{V}$ being the appropriate functional space. It can also be proved that, for this problem, the standard Galerkin discretization method provides a discrete irreductible problem which inherits the elliptic nature of the original problem. Therefore, the discrete solution $\mathbf{u}_{h} \in \mathcal{V}_{h}$ exists and it is unique, $\mathcal{V}_{h}$ being an appropriate finite element functional space. This situation is depicted in Figure 2.1 .

The situation is more complex when incompressibility takes places in the domain. Let us now consider a mixed problem in the continuum with some incompressibility constraint present, either globally or locally. It can be proved that 
CONTINUUM

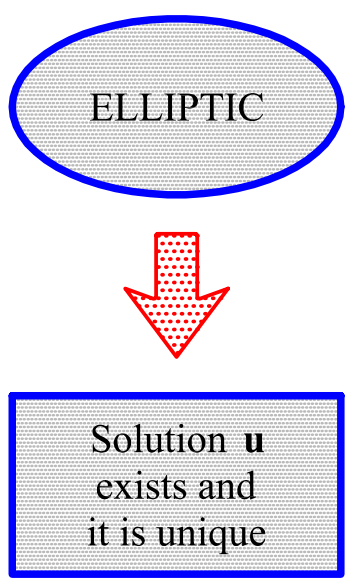

\section{DISCRETE}
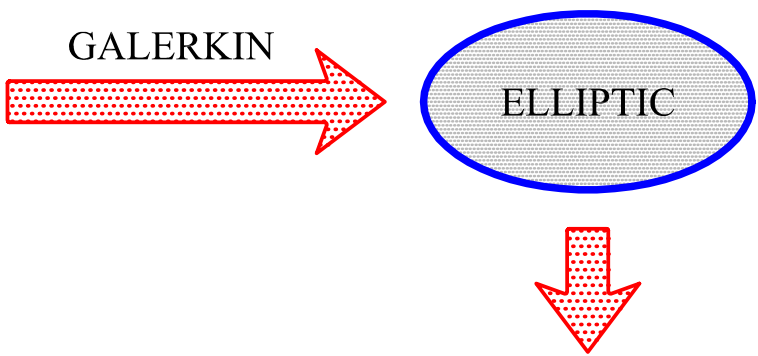

Solution $\mathbf{u}_{\mathbf{h}}$ exists and it is unique

Fig. 2.1: Continuum and discrete compressible irreductible elliptic problem

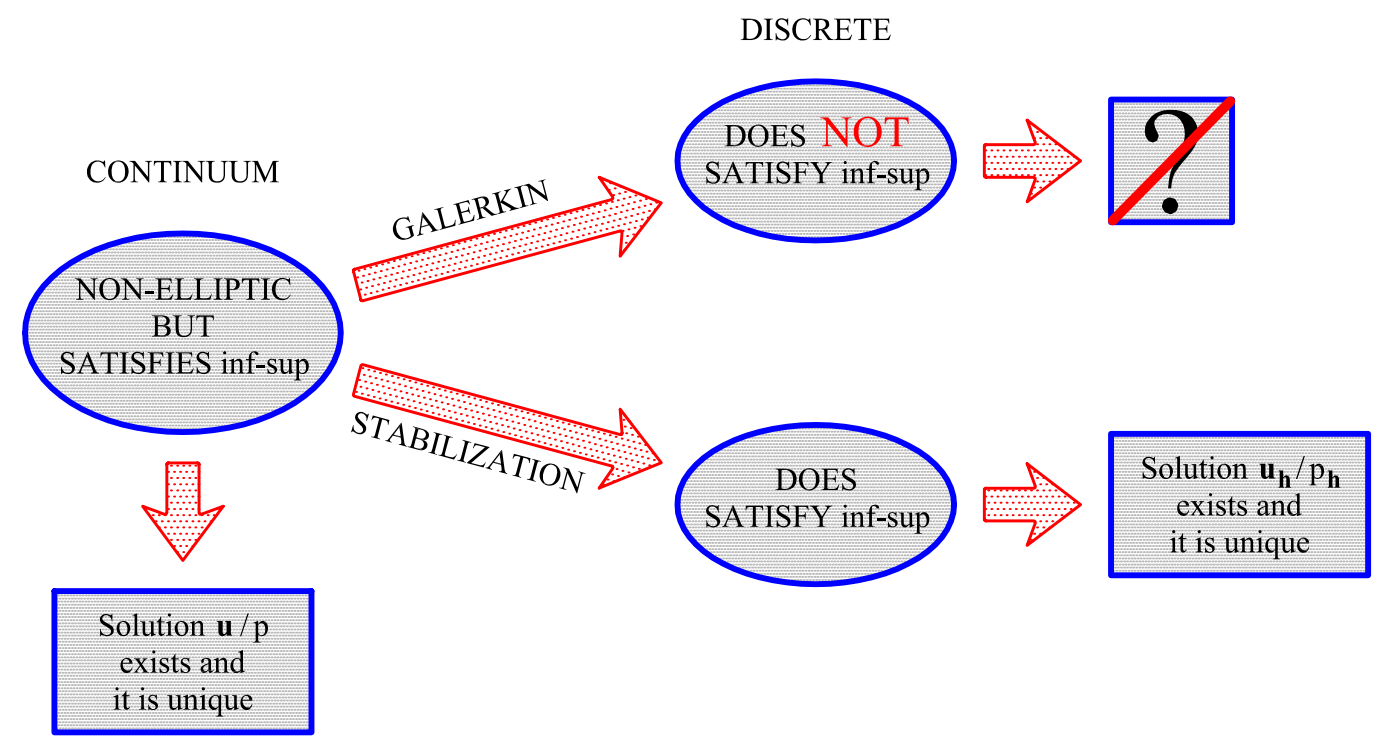

Fig. 2.2: Continuum and discrete incompressible mixed non-elliptic problem 
a solution $\mathbf{u} \in \mathcal{V}, p \in \mathcal{Q}$ exists and it is unique if the spaces $\mathcal{V}$ and $\mathcal{Q}$ satisfy the inf-sup condition [47]. Unfortunately, satisfaction of the necessary and sufficient inf-sup condition is not necessarily inherited by the corresponding discrete problem. For instance, if we attempt to solve the incompressible problem using standard simplexes, such as constant strain triangles or tetrahedra, we have $\mathcal{V}=P_{1}$ and $\mathcal{Q}=P_{0}$, which do not satisfy the inf-sup condition. This, and not the loss of ellipticity is the reason why the standard irreductible formulation fails miserably when attempting to solve localization problems. Figure 2.2 illustrates the explained situation for these problems.

It is, therefore, evident that the origin of the difficulties encountered when attempting to solve incompressible problems with non-stable finite element formulations does not lay on the local format of the constitutive equations, as many reputed researchers have assured during the last decade, but on the inadequacy of the discretization method used. It is also very clear that to solve localization problems it is necessary to use a discretization procedure that satisfies the necessary and sufficient inf-sup condition. In the following, we propose the use of the orthogonal sub-grid scale stabilization method (OSGS) to solve this type of problems.

\subsection{The Sub-Grid Scale approach}

The discrete finite element form of the problem is obtained from Eqs. (2.9)(2.10), substituting the displacement and pressure fields and their variations by their standard finite element interpolations:

$$
\begin{aligned}
\left(\nabla^{s} \mathbf{v}_{h}, \mathbf{s}_{h}\right)+\left(\nabla \cdot \mathbf{v}_{h}, p_{h}\right)-\left(\mathbf{v}_{h}, \mathbf{f}\right)-\left(\mathbf{v}_{h}, \overline{\mathbf{t}}\right)_{\partial \Omega} & =0 & \forall \mathbf{v}_{h} \\
\left(q_{h}, \nabla \cdot \mathbf{u}_{h}\right)-\left(q_{h}, \frac{1}{K} p_{h}\right) & =0 & \forall q_{h}
\end{aligned}
$$

where $\mathbf{u}_{h}, \mathbf{v}_{h} \in \mathcal{V}_{h}$ and $p_{h}, q_{h} \in \mathcal{Q}_{h}$ are the discrete displacement and pressure fields and their variations, defined onto the finite element spaces $\mathcal{V}_{h}$ and $\mathcal{Q}_{h}$, respectively.

As it is well known, the inf-sup condition [47], poses severe restrictions on the choice of the spaces $\mathcal{V}_{h}$ and $\mathcal{Q}_{h}$ when using the standard Galerkin discrete form (2.14)-(2.15). For instance, standard mixed elements with continuous equal order linear/linear interpolation for both fields are not stable, and the lack of stability 
shows as uncontrollable oscillations in the pressure field that usually, and very particularly in non linear problems, pollute the solution entirely. Fortunately, the strictness of the inf-sup condition can be circumvented by modifying the discrete variational form appropriately, in order to attain the necessary stability with the desired choice of interpolation spaces.

The basic idea of the sub-grid scale approach [31] is to consider that the continuous displacement field can be split in two components, one coarse and a finer one, corresponding to different scales or levels of resolution. The solution of the continuous problem contains components from both scales. For the solution of the discrete problem to be stable it is necessary to, somehow, include the effect of both scales in the approximation. The coarse scale can be appropriately solved by a standard finite element interpolation, which however cannot solve the finer scale. Nevertheless, the effect of this finer scale can be included, at least locally, to enhance the stability of the pressure in the mixed formulation.

To this end, the displacement field of the mixed problem will be approximated as

$$
\mathbf{u}=\mathbf{u}_{h}+\widetilde{\mathbf{u}}
$$

where $\mathbf{u}_{h} \in \mathcal{V}_{h}$ is the displacement component of the (coarse) finite element scale and $\widetilde{\mathbf{u}} \in \widetilde{\mathcal{V}}$ is the enhancement of the displacement field corresponding to the (finer) sub-grid scale. Let us also consider the corresponding variations $\mathbf{v}_{h} \in \mathcal{V}_{h}$ and $\widetilde{\mathbf{v}} \in \widetilde{\mathcal{V}}$, respectively. This extends the displacement solution space to $\mathcal{V} \simeq \mathcal{V}_{h} \oplus \widetilde{\mathcal{V}}$

It is reasonable to assume that the sub-grid displacements $\widetilde{\mathbf{u}}$ will be sufficiently "small" compared to $\mathbf{u}_{h}$; they can be viewed as a "high frequency" perturbation of the finite element field, which cannot be resolved in $\mathcal{V}_{h}$. It can also be assumed that $\widetilde{\mathbf{u}}$ and $\widetilde{\mathbf{v}}$ vanish on the boundary $\partial \Omega$. It must be pointed out that no sub-grid scale contribution has been considered on the pressure field.

Considering the subscales, the deviatoric stresses can be decomposed into two different contributions

$$
\mathbf{s}(\mathbf{u})=\mathbf{s}_{h}(\mathbf{u})+\widetilde{\mathbf{s}}(\mathbf{u})
$$

Notice that the stresses $\mathbf{s}$ are not a linear function of $\mathbf{u}$ and, therefore, it is not exactly true that $\mathbf{s}_{h}=\mathbf{s}_{h}\left(\mathbf{u}_{h}\right)$ and $\widetilde{\mathbf{s}}=\widetilde{\mathbf{s}}(\widetilde{\mathbf{u}})$. However, we can approximate the stresses corresponding to the coarse finite element scale in the standard fashion, as:

$$
\mathbf{s}_{h}(\mathbf{u}) \cong \mathbf{s}_{h}\left(\mathbf{u}_{h}\right)=2 G^{*} \mathbf{e}_{h}
$$


which can be computed following the integration algorithm proposed in the next chapter. In Eq. (2.18) we have introduced the secant shear modulus as (half) the ratio between the norms of the deviatoric stress and total strain tensors, $2 G^{*}=\left\|\mathbf{s}_{h}\right\| /\left\|\mathbf{e}_{h}\right\|$. For non-linear constitutive models, this ratio is obviously non-constant and it varies along the deformation process.

Being the enhancement $\widetilde{\mathbf{u}}$ "small" compared to $\mathbf{u}_{h}$, it is possible to make the following approximation for the strains due to the sub-scale:

$$
\frac{\|\widetilde{\mathbf{s}}\|}{\|\widetilde{\mathbf{e}}\|} \cong \frac{\left\|\mathbf{s}_{h}\right\|}{\left\|\mathbf{e}_{h}\right\|}=2 G^{*}
$$

which motivates the approximation:

$$
\widetilde{\mathbf{s}}(\mathbf{u}) \cong \widetilde{\mathbf{s}}(\widetilde{\mathbf{u}})=2 G^{*} \widetilde{\mathbf{e}}
$$

It can be remarked that the formalism of approximation (2.20) holds for other non-linear constitutive models, such as nonlinear elasticity or plasticity.

With these definitions, the discrete problem corresponding to Eqs. (2.7) and (2.8) is now:

$$
\begin{array}{rlrl}
\left(\nabla^{s} \mathbf{v}_{h}, \mathbf{s}_{h}\right)+\left(\nabla^{s} \mathbf{v}_{h}, \widetilde{\mathbf{s}}\right)+\left(\nabla \cdot \mathbf{v}_{h}, p_{h}\right) & & \\
-\left(\mathbf{v}_{h}, \mathbf{f}\right)-\left(\mathbf{v}_{h}, \overline{\mathbf{t}}\right)_{\partial \Omega_{t}} & =0 & & \forall \mathbf{v}_{h} \\
\left(\widetilde{\mathbf{v}}, \nabla \cdot \mathbf{s}_{h}\right)+(\widetilde{\mathbf{v}}, \nabla \cdot \widetilde{\mathbf{s}})+\left(\widetilde{\mathbf{v}}, \nabla p_{h}\right)+(\widetilde{\mathbf{v}}, \mathbf{f}) & =0 & \forall \widetilde{\mathbf{v}} \\
\left(q_{h}, \nabla \cdot \mathbf{u}_{h}\right)+\left(q_{h}, \nabla \cdot \widetilde{\mathbf{u}}\right) & =0 & \forall q_{h}
\end{array}
$$

where Eq. (2.21) has been integrated by parts, recalling that $\widetilde{\mathbf{u}}$ and $\widetilde{\mathbf{v}}$ vanish on the boundary.

Due to the approximation used, Eq. (2.16), and the linear independence of $\mathbf{v}_{h}$ and $\widetilde{\mathbf{v}}$, Eq. (2.16), now the continuum Eq. (2.10) unfolds in two discrete equations, one related to each scale considered. Equations (2.21) and (2.23) are defined in the finite element spaces $\mathcal{V}_{h}$ and $\mathcal{Q}_{h}$, respectively. The first one solves the balance of momentum including a stabilization term $S_{1}=\left(\nabla^{s} \mathbf{v}_{h}, \widetilde{\mathbf{s}}\right)$ depending on the sub-grid stresses $\widetilde{\mathbf{s}}$. The second one enforces the incompressibility condition including a stabilization term $\mathrm{S}_{2}=\left(q_{h}, \nabla \cdot \widetilde{\mathbf{u}}\right)$ depending on the sub-grid displacements $\widetilde{\mathbf{u}}$. On the other hand, equation (2.22) is defined in the sub-grid scale space $\widetilde{\mathcal{V}}$ and, hence, it cannot be solved by the finite element mesh. Let us now rewrite Eq. (2.22) in the form:

$$
-(\widetilde{\mathbf{v}}, \nabla \cdot \widetilde{\mathbf{s}})=\left(\widetilde{\mathbf{v}}, \mathbf{r}_{h}\right) \quad \forall \widetilde{\mathbf{v}} \in \widetilde{\mathcal{V}}
$$


where the residual of the Cauchy equation in the finite element scale is defined as:

$$
\mathbf{r}_{h}=\mathbf{r}_{h}\left(\mathbf{u}_{h}, p_{h}\right)=\nabla \cdot \mathbf{s}_{h}+\nabla p_{h}+\mathbf{f}
$$

Now, some appropriate approximations are necessary. According to hypotheses (2.19) and (2.20), let us assume that, within each finite element $\Omega_{e}$,

$$
\|\nabla \cdot \widetilde{\mathbf{s}}\| \cong \frac{\|\widetilde{\mathbf{s}}\|}{c_{1} h_{e}}=\frac{2 G_{e}^{*}\|\widetilde{\mathbf{e}}\|}{c_{1} h_{e}} \cong \frac{2 G_{e}^{*}}{c_{1} h_{e}}\left(\frac{\|\widetilde{\mathbf{u}}\|}{c_{2} h_{e}}\right)=\frac{1}{\tau_{e}}\|\widetilde{\mathbf{u}}\|
$$

where the parameter $\tau_{e}=c h_{e}^{2} / 2 G_{e}^{*}$ is defined as a function of the characteristic length of the element $h_{e}$ and the current secant shear modulus $G_{e}^{*}$. The constant $c=c_{1} c_{2}=\mathcal{O}(1)$ has to be determined through numerical testing. This very simple and heuristic approximation of the effect of the sub-scale is one of the keys to the success of the stabilization procedure of the incompressible problem. More elaborated assumptions may be needed in other situations.

Integrating the stabilization terms by parts, and recalling that the subscale displacements vanish on the boundary, it yields

$$
\begin{aligned}
& \mathrm{S}_{1}=\left(\nabla^{s} \mathbf{v}_{h}, \widetilde{\mathbf{s}}\right)=-\left(\widetilde{\mathbf{u}}, \nabla \cdot\left(2 G^{*} \operatorname{dev} \nabla^{s} \mathbf{v}_{h}\right)\right) \\
& \mathrm{S}_{2}=\left(q_{h}, \nabla \cdot \widetilde{\mathbf{u}}\right)=-\left(\widetilde{\mathbf{u}}, \nabla q_{h}\right)
\end{aligned}
$$

The term $\nabla \cdot\left(\operatorname{dev} \nabla^{s} \mathbf{v}_{h}\right)$ in $(2.27)$ involves second derivatives of finite element functions which vanish when linear elements are used. In the case of higher order elements these derivatives can be neglected, leading to a method which is still consistent, but with a non-optimal rate of convergence [39].

\subsection{Orthogonal Sub-Grid Scales (OSGS) and Galerking Least Squares (GLS) stabilizations}

The objective in this section is to obtain a useful expression for the sub-grid scale displacements $\widetilde{\mathbf{u}}$ to be introduced in the stabilization term $\mathrm{S}_{2}$ in Eq. (2.23).

It was argued in [32] that a very natural choice for the unknown subgrid space $\widetilde{\mathcal{V}}$ is the space orthogonal to the finite element space, referred to hereafter as $\mathcal{V}_{h}^{\perp}$. This means approximating the displacement solution space as $\mathcal{V} \simeq \mathcal{V}_{h} \oplus \mathcal{V}_{h}^{\perp}$. The subsequent stabilization method is called orthogonal sub-grid scale method, and it has already been successfully applied to several problems in fluid mechanics. 
Also, the format of the Eq. (2.24), which is exact for the fine scale and nonlocal, strongly suggests that $\nabla \cdot \widetilde{\mathbf{s}}$, and hence $\widetilde{\mathbf{u}}$, are driven by the residual of the coarse scale, $\mathbf{r}_{h}$. In [38] it is reasoned that the sought effect of the finer scale is to explicitly account for the distributional effects of the residual of the coarse scale.

Because of these two reasons, we will take $\widetilde{\mathbf{v}} \in \mathcal{V}_{h}^{\perp}$, and assume that

$$
-(\widetilde{\mathbf{v}}, \nabla \cdot \widetilde{\mathbf{s}}) \cong\left(\widetilde{\mathbf{v}}, P_{h}^{\perp}\left(\mathbf{r}_{h}\right)\right) \quad \forall \widetilde{\mathbf{v}} \in \mathcal{V}_{h}^{\perp}
$$

where $P_{h}^{\perp}(\mathbf{x})$ is the orthogonal projection of $\mathbf{x}$ onto $\mathcal{V}_{h}^{\perp}$, which can be expressed as $P_{h}^{\perp}(\mathbf{x})=\mathbf{x}-P_{h}(\mathbf{x})$. The $L^{2}$ projection of $\mathbf{x}$ onto the finite element space, or least square fitting, can easily be computed from the orthogonality condition

$$
\left(P_{h}(\mathbf{x})-\mathbf{x}, \boldsymbol{\eta}_{h}\right)=0 \quad \forall \boldsymbol{\eta}_{h} \in \mathcal{V}_{h}
$$

Using approximations (2.26) and (2.29), the sub-scale displacements can be localized within each finite element $\Omega_{e}$, and be expressed as

$$
\widetilde{\mathbf{u}}_{e}=\tau_{e} P_{h}^{\perp}\left(\mathbf{r}_{h}\right) \quad \in \mathcal{V}_{h}^{\perp}
$$

where the positive sign is necessary from stability considerations.

Some remarks are in order:

1. As expected, $\widetilde{\mathbf{u}}$ is sufficiently "small" compared to $\mathbf{u}_{h}\left(\widetilde{\mathbf{u}} \simeq \mathcal{O}\left(h^{2}\right)\right)$.

2. With this definition, $\widetilde{\mathbf{u}}$ is discontinuous across element boundaries. For linear elements, $\widetilde{\mathbf{u}}$ is piece-wise linear.

3. Even if defined element-wise, $\widetilde{\mathbf{u}}_{e}$ cannot be condensed at element level, because $P_{h}^{\perp}(\cdot)$ is a global operator.

4. In the localization process, it is necessary to neglect the integrals over element faces involving the sub-scale, in front of the integrals over the element volumes. This is justified in [39] resorting to Fourier analysis and recalling that the subscale is associated to higher frequencies that the grid scale. It is worth to mention that for "bubble"-type enhancements these boundary terms are null by construction [40], [41]. 
5. Eq. (2.31) does not need to be interpreted point-wise, as the values of $\widetilde{\mathbf{u}}$ are not used in the stabilization procedure; only the integrals $S_{1}$ and $S_{2}$ in Eqs. (2.27)-(2.28) are needed.

It must be pointed out that $\mathbf{f}$ in (2.25) can be assumed to belong to the space $\mathcal{V}_{h}$ and, consequently, $P_{h}^{\perp}(\mathbf{f})=\mathbf{0}$. Also, $\nabla \cdot \mathbf{s}_{h}$ in $(2.25)$ involves second derivatives of finite element functions which vanish when linear elements are used. Taking all this into account, expression (2.31) transforms in

$$
\widetilde{\mathbf{u}}_{e}=\tau_{e}\left(\nabla p_{h}-P_{h}\left(\nabla p_{h}\right)\right)
$$

Finally, substituting Eq. (2.32) into the expression of the stabilization term $\mathrm{S}_{2}$, see Eq. (2.28), it simplifies as

$$
\mathrm{S}_{2}=-\sum_{e=1}^{n_{\text {elm }}} \tau_{e}\left(\nabla q_{h} \cdot\left[\nabla p_{h}-P_{h}\left(\nabla p_{h}\right)\right]\right)
$$

Observe that this stabilization term is computed in an element by element manner and, within each element, its magnitude depends on the difference between the continuous (projected) and the discontinuous (elemental) pressure gradient. This means that the term added to secure a stable solution decreases very rapidly upon mesh refinement, as the finite element scale becomes finer and the projection of the residual reduces. This happens at a greater rate than with other stabilization techniques, such as the Galerkin Least Square $(G L S)$ method, where the stabilization terms are proportional to the residual itself, see [42], [43] and below.

The projection of the pressure gradient onto the finite element space $\mathcal{V}_{h}, \boldsymbol{\Pi}_{h}=$ $P_{h}\left(\nabla p_{h}\right)$, is computed as:

$$
\left(\nabla p_{h}, \boldsymbol{\eta}_{h}\right)=\left(\boldsymbol{\Pi}_{h}, \boldsymbol{\eta}_{h}\right) \quad \forall \boldsymbol{\eta}_{h} \in \mathcal{V}_{h}
$$

As a result of the above procedure, the stabilized mixed system of equations proposed in this work to solve the problem of incompressible elasto-damage behavior with linear/linear interpolations for the displacement and pressure fields is the following:

$$
\begin{array}{rlrl}
\left(\nabla^{s} \mathbf{v}_{h}, \mathbf{s}_{h}\right)+\left(\nabla \cdot \mathbf{v}_{h}, p_{h}\right)-\left(\mathbf{v}_{h}, \mathbf{f}\right)-\left(\mathbf{v}_{h}, \overline{\mathbf{t}}\right)_{\partial \Omega_{t}} & =0 & & \forall \mathbf{v}_{h}(2.35) \\
\left(q_{h}, \nabla \cdot \mathbf{u}_{h}\right)-\left(q_{h}, \frac{1}{K} p_{h}\right)-\sum_{e=1}^{n_{e l m}} \tau_{e}\left(\nabla q_{h} \cdot\left[\nabla p_{h}-\mathbf{\Pi}_{h}\right]\right) & =0 & \forall q_{h}(2.36) \\
\left(\nabla p_{h}, \boldsymbol{\eta}_{h}\right)-\left(\boldsymbol{\Pi}_{h}, \boldsymbol{\eta}_{h}\right) & =0 & \forall \boldsymbol{\eta}_{h}(2.37)
\end{array}
$$


It is important to point out that, when using linear/linear displacement and pressure interpolations, the only stabilization term appears in the incompressibility equation (2.36), see [33], [34], [35]. Observe that in it, the third nodal variable

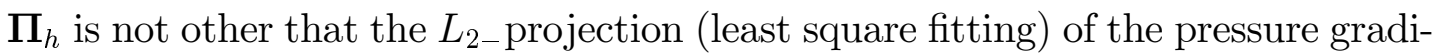
ent, $\boldsymbol{\Pi}_{h}=P_{h}\left(\nabla p_{h}\right)$. The next section shows that the drawback of accounting for an extra nodal variable can be easily overcome to achieve a robust and efficient procedure.

An alternative stabilization method is the one known as Galerkin Least Square $(G L S)$, originally proposed in [42]. The corresponding stabilized discrete problem reads:

$$
\begin{aligned}
& \left(\nabla^{s} \mathbf{v}_{h}, \mathbf{s}_{h}\right)+\left(\nabla \cdot \mathbf{v}_{h}, p_{h}\right)-\left(\mathbf{v}_{h}, \mathbf{f}\right)-\left(\mathbf{v}_{h}, \overline{\mathbf{t}}\right)_{\partial \Omega_{t}}=0 \quad \forall \mathbf{v}_{h} \\
& \left(q_{h}, \nabla \cdot \mathbf{u}_{h}\right)-\left(q_{h}, \frac{1}{K} p_{h}\right)-\sum_{e=1}^{n_{\text {elm }}} \tau_{e}\left(\nabla q_{h} \cdot \nabla p_{h}\right)=0 \quad \forall q_{h}
\end{aligned}
$$

which has a format very similar to the OSGS method, but does not require the computation of any extra nodal variable. Experience shows that the GLS method is more diffusive that the OSGS stabilization. This means that GLS is somewhat more "robust" than OSGS, but sometimes less sharp localizations are obtained.

\subsection{Stabilization parameter}

The stabilization techniques discussed in the previous section are designed to provide stability to the incompressible elasto-damage problem. In reference [35] it was found that, with perfect plasticity, the stabilization parameter has to be modified to account for the development of the plastic regime, as deformation localizes into weak discontinuities; otherwise, pressure oscillations arise in the vicinity of the damaged areas and pollute the solution. In [35], it was proposed to enhance the stabilization properties using a nonlinear stabilization parameter

$$
\tau_{e}=\frac{c h_{e}^{2}}{2 G^{*}}
$$

computed as a function of the characteristic length of the element $h_{e}$ and the current secant shear modulus $G^{*}$, defined as (half) the ratio between the norms of the deviatoric stress and total strain tensors, $2 G^{*}=\|\mathbf{s}\| /\|\mathbf{e}\|$. For nonlinear constitutive models, this ratio is obviously non-constant and it varies along the 
deformation process. In the softening regime, it is clear that, as deformation evolves, the secant modulus $G^{*}$ decreases and, consequently, the value of $\tau_{e}$ increases, further relaxing the incompressibility constraint. The constant $c=\mathcal{O}(1)$ has to be determined through numerical testing.

It is obvious in Eqs. (2.36) and (2.39) that the effect of the stabilization technique is to add a term that relaxes the strict quasi-incompressibility constraint posed by this equation. Note that this relaxation is very small, as $\tau_{e}=\mathcal{O}\left(h_{e}^{2}\right)$ and it depends on the difference between the discontinuous pressure gradient and its continuous least square projection. This difference tends to vanish at a fast rate as the mesh is refined.

\subsection{Consistent residual viscosity}

For J2-softening materials, shear strain localizes and the modulus $G^{*}$ decreases very fast with increasing deformation and, ultimately, vanishes, yielding very large values of the stabilization parameter. At the same time, the components of the tangent deviatoric constitutive tensor diminish, eventually leading to a non-positive definite global stiffness matrix. It is found that these circumstances cause numerical difficulties that translate in slow or even lack of convergence of the solution of the nonlinear discrete equations, particularly in problems involving singular points, where the strains reach very large values.

One obvious way to alleviate this difficulty is to define an ad hoc cut-off value for the decrease of the values of the secant modulus $G^{*}$ and, consequently, on the increase of the values of the stabilization parameter $\tau_{e}$. Unfortunately, experience shows that this leads to the impossibility of the total elimination of the local oscillations of the pressure. The greater the value of the cut-off, the better is the convergence, but greater are the remaining local pressure oscillations.

Therefore, a different approach is preferred to enhance the convergence of the nonlinear equilibrium iterations. This consists in introducing a consistent residual viscous regularization of the damage model. The numerical benefits of viscous regularizations are well known and documented in the literature, but it is also true that the addition of "artificial" viscosity in an indiscriminate fashion rather changes the nature of the obtained solution. It is therefore adamant to devise a high order scheme where the minimal amount of artificial viscosity is added only where it is needed and, also, in a consistent manner, that is, with values tending 
to zero for decreasing element sizes.

It is here proposed to regularize the elasto-damage constitutive model with a residual artificial viscosity defined in terms of the orthogonal projection of the residual of the momentum equation onto the finite element space (see [35] and $[36])$, in the form

$$
\vartheta=c^{\prime} \frac{h_{e} \Delta t}{G}\left\|\nabla p_{h}-\Pi_{h}\right\|
$$

where $c^{\prime}=\mathcal{O}(1)$ is a constant, $h_{e}$ is the characteristic length of the element, $\Delta t$ is the time step size and $\sigma_{o}$ is the uniaxial strength. Note that as it is defined, $\vartheta$ has units of time.

Note that this viscosity acts only in those elements where the momentum equation is not exactly satisfied and that, for linear simplex, it is $\vartheta=\mathcal{O}\left(h_{e}^{2} \Delta t\right)$. This means that it maintains the order of the finite element approximation, as it vanishes upon mesh (and time increment) refinement with the appropriate rate.

The structure of expression (2.41) suggests that it is also possible to define the viscosity purely in terms of the norm of the pressure gradient, in the form

$$
\vartheta=c^{\prime} \frac{h_{e} \Delta t}{G}\left\|\nabla p_{h}\right\|
$$

This second proposal is consistent with the definition of the stabilization term used in the original GLS method.

\subsection{Implementation and computational aspects}

Due to the nonlinear dependence of the stresses on the displacements, the solution of the system of equations (2.35)-(2.37) requires the use of an appropriate incremental/iterative procedure such as the Newton-Raphson method. Within such a procedure, the system of linear equations to be solved for the $(i+1)$-th equilibrium iteration of the $(n+1)$-th time (or load) step is:

$$
\left[\begin{array}{ccc}
\mathbf{K}_{\mathrm{dev}} & \mathbf{G} & \mathbf{0} \\
\mathbf{G}^{T} & -\left(\frac{1}{K} \mathbf{M}+\mathbf{L}_{\boldsymbol{\tau}}\right) & \mathbf{G}_{\boldsymbol{\tau}}^{T} \\
\mathbf{0} & \mathbf{G}_{\boldsymbol{\tau}} & -\mathbf{M}_{\boldsymbol{\tau}}
\end{array}\right]^{(n+1, i)}\left[\begin{array}{l}
\delta \mathbf{U} \\
\delta \mathbf{P} \\
\delta \mathbf{\Pi}
\end{array}\right]^{(n+1, i+1)}=-\left[\begin{array}{l}
\mathbf{R}_{1} \\
\mathbf{R}_{2} \\
\mathbf{0}
\end{array}\right]^{(n+1, i)}
$$

where $\delta \mathbf{U}, \delta \mathbf{P}$ and $\delta \boldsymbol{\Pi}$ are the iterative corrections to the nodal values for the displacements, pressure and pressure gradient, respectively, $\mathbf{R}_{1}$ and $\mathbf{R}_{2}$ are the resid- 
ual vectors associated to the satisfaction of the balance of momentum and incompressibility equations, respectively, and the global matrices $\mathbf{K}_{\mathrm{dev}}, \mathbf{G}, \mathbf{G}_{\tau}, \mathbf{M}, \mathbf{L}_{\tau}$ and $\mathbf{M}_{\boldsymbol{\tau}}$ come from the standard assembly procedure of the elemental contributions, for the $i$-th iteration of the $n+1$ step. Note that this global matrix is symmetric, but it is not positive definite. Each one of the elemental matrices $\mathbf{K}^{(e)}$ has the symmetric structure:

$$
\left[\mathbf{K}^{A B}\right]^{(e)}=\left[\begin{array}{ccc}
\mathbf{K}_{\mathrm{dev}}^{A B} & \mathbf{G}^{A B} & \mathbf{0} \\
\left(\mathbf{G}^{A B}\right)^{T} & -\tau_{e} L^{A B} & \tau_{e}\left(\mathbf{G}^{A B}\right)^{T} \\
\mathbf{0} & \tau_{e} \mathbf{G}^{A B} & -\tau_{e} \mathbf{M}^{A B}
\end{array}\right]^{(e)}
$$

where the entry $(\cdot)^{A B}$ is a sub-matrix corresponding to the local nodes $A$ and $B$. In equation (2.44), $\mathbf{K}_{\mathrm{dev}}^{A B}$ is the deviatoric component of the standard elastodamaged tangent stiffness matrix defined as:

$$
\mathbf{K}_{\mathrm{dev}}^{A B}=\int_{\Omega_{e}} \mathbf{B}_{A}^{T} \mathbf{D}_{\mathrm{dev}}^{e p} \mathbf{B}_{B} d \Omega
$$

where $\mathbf{D}_{\text {dev }}^{e p}$ is the deviatoric constitutive matrix and $\mathbf{B}$ is a standard deformation sub-matrix. The generic term of the discrete gradient matrix operator $\mathbf{G}^{A B}$ is given by:

$$
\mathbf{G}^{A B}=\int_{\Omega_{e}}\left[\nabla N^{A}\right] N^{B} d \Omega \quad, \quad \text { where }\left[\nabla N^{A}\right]=\left[N_{, x}^{A} N_{, y}^{A} N_{, z}^{A}\right]^{T}
$$

while the laplacian term $L^{A B}$ can be expressed as:

$$
L^{A B}=\int_{\Omega_{e}}\left[\nabla N^{A}\right]^{T}\left[\nabla N^{B}\right] d \Omega
$$

Finally, $\mathbf{M}^{A B}$ is the "mass" matrices associated to the displacement field:

$$
\mathbf{M}^{A B}=\int_{\Omega_{e}} \mathbf{N}^{A} \mathbf{N}^{B} d \Omega
$$

The expensive monolithic solution of system (2.43) can be avoided by using an iterative procedure, in which the pressure projection $\Pi^{(n+1, i+1)}$ is solved independently and explicitly. To this end, from the third equation, it is possible to express $\boldsymbol{\Pi}^{(n+1, i+1)}$ in terms of $\mathbf{P}^{(n+1, i+1)}$ as:

$$
\boldsymbol{\Pi}^{(n+1, i+1)}=\left(\mathbf{M}_{\boldsymbol{\tau}}^{-1}\right)^{(n+1, i)} \mathbf{G}_{\boldsymbol{\tau}}{ }^{(n+1, i)} \mathbf{P}^{(n+1, i+1)} \cong \overline{\mathbf{M}}^{-1} \mathbf{G} \mathbf{P}^{(n+1, i+1)}
$$


The computation of the projections $\boldsymbol{\Pi}$ can be transformed in a straight-forward operation by neglecting the difference in the $\tau_{e}$ coefficient in adjacent elements and considering an approximate lumped mass matrix $\overline{\mathbf{M}}$.

One further approximation can be introduced to make the solution of the mixed system of equations more efficient from the computational point of view. This consists in an staggered scheme, keeping the projected pressure gradient constant during the equilibrium iterations within each time increment, taking it equal to the corresponding value at the end of the previous time step, that is $\boldsymbol{\Pi}^{(n+1, i+1)} \cong \boldsymbol{\Pi}^{(n)}$. This strategy has proved effective without loss of precision nor robustness.

From the above, the implementation of the GLS method is straight-forward; it is enough to delete the third equation in (2.43), together with the corresponding third column of the global matrix, to yield:

$$
\left[\begin{array}{cc}
\mathbf{K}_{\mathrm{dev}} & \mathbf{G} \\
\mathbf{G}^{T} & -\left(\frac{1}{K} \mathbf{M}+\mathbf{L}_{\boldsymbol{\tau}}\right)
\end{array}\right]^{(n+1, i)}\left[\begin{array}{l}
\delta \mathbf{U} \\
\delta \mathbf{P}
\end{array}\right]^{(n+1, i+1)}=-\left[\begin{array}{l}
\mathbf{R}_{1} \\
\mathbf{R}_{2}
\end{array}\right]^{(n+1, i)}
$$

where the effect of the stabilization matrix $\mathbf{L}_{\tau}$ is evident. For the OSGS, the stabilization matrix can be formally expressed as $\widehat{\mathbf{L}}_{\tau}=\mathbf{L}_{\tau}-\mathbf{G}_{\tau}^{T} \mathbf{M}_{\tau}^{-1} \mathbf{G}_{\tau}$. 


CHAPTEA 2. BOUNDATY TALUE PROBLEM




\section{Mixed formulation for J2 damage}

\subsection{Constitutive Equation}

The Continuum Damage Mechanics Theory (CDMT) is based on the definition of the effective stress concept, which is introduced in connection with the hypothesis of strain equivalence [51]: the strain associated with a damaged state under the applied stress $\sigma$ is equivalent to the strain associated with its undamaged state under the effective stress $\bar{\sigma}$. In the present work, the effective deviatoric stress tensor $\overline{\mathbf{s}}$ will assume the following form:

$$
\overline{\mathbf{s}}=2 G \mathbf{e}
$$

The constitutive equation for the J2-damage model is defined as:

$$
\begin{aligned}
p & =K \varepsilon_{v} \\
\mathbf{s} & =(1-d) \overline{\mathbf{s}}=(1-d) 2 G \mathbf{e}
\end{aligned}
$$

where we have introduced one internal-like variable, $d$, the damage index, whose definition and evolution is given below.

\subsection{Characterization of Damage}

In order to clearly define concepts such as loading, unloading, or reloading for general 3D stress states, a scalar positive quantity, termed as equivalent stress, is defined. This allows the comparison of different 2D and 3D stress states. With such a definition, distinct 3D stress states can be mapped to a single equivalent $1 D$ shear test, which makes their quantitative comparison possible [52], [53]. 
In the present work, the equivalent stress will assume the following form:

$$
\tau=\sqrt{\frac{3}{2}}\|\overline{\mathbf{s}}\|=\sqrt{\frac{3}{2}}[\overline{\mathbf{s}}: \overline{\mathbf{s}}]^{1 / 2}
$$

which corresponds to the usual definition of the equivalent von Mises stress $\bar{s}$.

With the above definition for the equivalent effective stress, the damage criterion, $\Phi$, is introduced as:

$$
\Phi(\tau, r)=\tau-r=\bar{s}-r \leq 0
$$

Variable $r$ is an internal stress-like variable that is interpreted as the current damage threshold, in the sense that its value controls the size of the (monotonically) expanding damage surface. The initial value of the damage threshold is $r_{o}=\sigma_{o}$, where $\sigma_{o}$ is the initial uniaxial damage stress.

Note that the damage criterion is defined in the effective deviatoric stress space (or, alternatively, in the deviatoric strain space). In the principal stress space, the shape of the damage criterion is defined by the well-known von Mises cylinder with axis along the hydrostatic axis. Figure 3.1a shows a schematic representation of this damage criterion.

The evolution (expansion) of the damage bounding surface in the normalized space for loading, unloading and reloading conditions is controlled by the KuhnTucker relations and the damage consistency condition, which are

$$
\dot{r} \geq 0 \quad \Phi(\tau, r) \leq 0 \quad \dot{r} \Phi(\tau, r)=0
$$

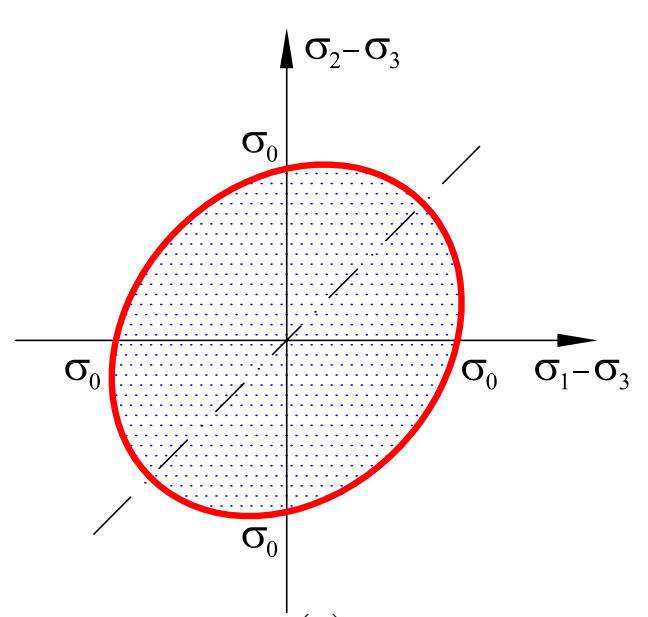

(a)

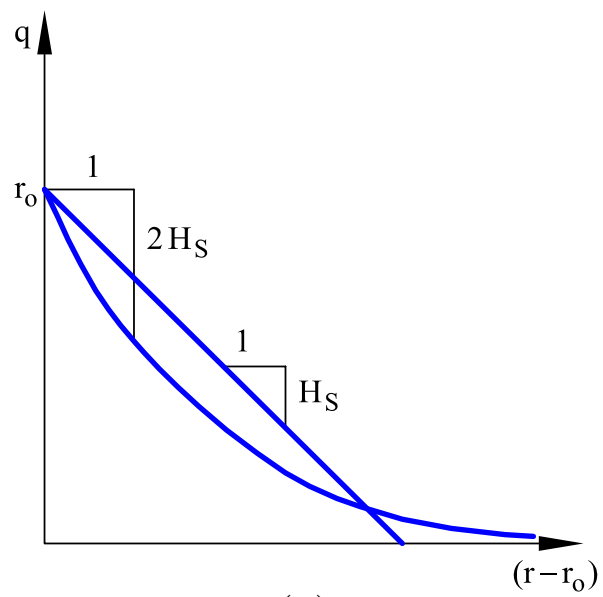

(b)

Fig. 3.1: J2-damage model: (a) damage surface, (b) softening functions 


$$
\text { if } \Phi(\tau, r)=0 \text { then } \dot{r} \dot{\Phi}(\tau, r)=0
$$

leading, in view of Eq. (3.5), to the loading condition

$$
\dot{r}=\dot{\tau}
$$

This, in turn, leads to the explicit definition of the current values of the internal variable $r$ in the form

$$
r=\max \left\{r_{0}, \max (\tau)\right\}
$$

Note that Eq. (3.8) allows to compute the current values for $r$ in terms of the current value of $\tau$, which depends explicitly on the current deviatoric strains (see Eqs. (3.36) and (3.4)).

Finally, the damage index $d=d(r)$ is explicitly defined in terms of the corresponding current value of the damage threshold, so that it is a monotonically increasing function such that $0 \leq d \leq 1$.

In this work, we will use the following functions:

- Linear softening:

$$
d(r)=\left\{\begin{array}{cl}
\left(1+H_{S}\right)\left(1-\frac{r_{0}}{r}\right) & r_{0} \leq r \leq r_{u}=r_{0}\left(1+\frac{1}{H_{S}}\right) \\
1 & r_{u} \geq r
\end{array}\right.
$$

- Exponential softening:

$$
d(r)=1-\frac{r_{0}}{r} \exp \left\{-2 H_{S}\left(\frac{r-r_{0}}{r_{0}}\right)\right\} \quad r_{0} \leq r
$$

where $H_{S} \geq 0$ is a constant.

It is also possible to express the damage laws in the form:

$$
d(r)=1-\frac{q(r)}{r} \quad r_{0} \leq r
$$

where the function $q=q(r)=(1-d(r)) r$ is the stress-like softening function.

In this format, the softening laws can be rewritten as

- Linear softening:

$$
d(r)=\left\{\begin{array}{cl}
\left(1+H_{S}\right)\left(1-\frac{r_{0}}{r}\right) & r_{0} \leq r \leq r_{u}=r_{0}\left(1+\frac{1}{H_{S}}\right) \\
1 & r_{u} \geq r
\end{array}\right.
$$


- Exponential softening:

$$
q(r)=r_{0} \exp \left\{-2 H_{S}\left(\frac{r-r_{0}}{r_{0}}\right)\right\} \quad r_{0} \leq r
$$

Figure $3.1 \mathrm{~b}$ shows a schematic representation of both these functions.

\subsection{Rate dependent damage}

In many materials there is a strong coupling between nonlinear rate-sensitivity and damage growth. Therefore, it is natural to develop a rate dependent constitutive model within the framework of CDMT, evolving from the above presented rate independent damage model, and which accounts for strain rate dependency via the damage evolution laws [45].

To this end, let us consider a viscous regularization of the rate-independent damage threshold evolution law defined by Eq. (3.7), so that this is replaced by:

$$
\dot{r}=\frac{\langle\tau-r\rangle}{\vartheta}
$$

where $\langle\cdot\rangle$ are the Macaulay brackets and $\vartheta$ is the retardation time for damage.

Note that this modification of the evolution law only affects the integration of the damage threshold $r$, but not the damage variable $d$ itself. This is still obtained in a closed form, through the explicit definition of the function $d=$ $d(r)$. Additionally, it is worth to remark that Eq. (3.14) guarantees monotonic increasing of the damage threshold, and, therefore, also of the damage index $(\dot{d} \geq 0)$.

It must be noted that the definition of the evolution law (3.14) replaces the Kuhn-Tucker and consistency conditions, Eqs. (3.6a) and (3.6b), of the rate independent model.

The reasoning behind the above law is analogous to the classical Perzyna viscoplastic regularization often used in the framework of Computational Plasticity. It can be seen that this assumed rate dependent formulation is a general form for the evolution of the damage threshold, so that for a null value of the retardation damage parameter, the rate-independent (or inviscid) damage evolution law is recovered exactly, while for an infinite value of $\vartheta$, evolution of the damage variables is prevented, thus rendering an instantaneously elastic response. 
The rate-dependent model can also be written in a Duvaut-Lions format, as

$$
\dot{r}=\frac{\left\langle r_{\infty}-r\right\rangle}{\vartheta} \geq 0
$$

where $r_{\infty}$ is the value of the damage threshold corresponding to the current value of $\tau$ as $t / \vartheta \rightarrow \infty$. As the rate-dependent (viscous) model relaxes to the rateindependent (inviscid) solution, it is obvious that in the inviscid limit, satisfaction of the consistency condition ensures that $r_{\infty}=\tau$. Therefore, Eqs. (3.14) and (3.15) are completely equivalent.

\subsection{Thermodynamic Framework}

Let us define the elastic free energy in the form

$$
\begin{aligned}
W^{e} & =W_{\mathrm{vol}}\left(\varepsilon_{v}\right)+W_{\mathrm{dev}}^{e}(\mathbf{e}) \\
& =\frac{1}{2} K \varepsilon_{v}^{2}+\frac{1}{2}(2 G) \mathbf{e}: \mathbf{e} \geq 0
\end{aligned}
$$

The mechanical free energy term for the damage model is defined in the form:

$$
\begin{aligned}
W & =W_{\mathrm{vol}}\left(\varepsilon_{v}\right)+(1-d) W_{\mathrm{dev}}^{e}(\mathbf{e}) \\
& =\frac{1}{2} K \varepsilon_{v}^{2}+(1-d) \frac{1}{2}(2 G) \mathbf{e}: \mathbf{e} \geq 0
\end{aligned}
$$

From this, and recalling that $0 \leq d \leq 1$, it is obvious that $W \geq 0$.

The constitutive equation for the damage model is obtained using Coleman's method as:

$$
\begin{aligned}
p & =\partial_{\varepsilon_{v}} W=K \varepsilon_{v} \\
\mathbf{s} & =\partial_{\mathbf{e}} W=(1-d) 2 G \mathbf{e}
\end{aligned}
$$

The mechanical dissipation can be expressed as

$$
\dot{\mathcal{D}}=W_{\mathrm{dev}}^{e} \dot{d} \quad \geq 0
$$

provided that the damage index increases monotonically, $\dot{d} \geq 0$. 


\subsection{Softening behaviour and regularization}

Expressions (3.9) and (3.10) are able to reproduce the softening branch that occurs in a 1D shear test after the peak stress is reached, with the stress decreasing asymptotically to the strain axis. With these evolution laws for $d$, a finite area is retained between the stress-strain curve and the strain axis. This area defines the available energy to be dissipated in the control volume.

In finite element analysis this entails the loss of objectivity of the results, in the sense that the inelastic strains tend to localize in a band that is only one element across, independently of the element size $h_{e}$. Upon mesh refinement, as $h_{e}$ tends to zero, strains tend to concentrate on a band of zero thickness (a geometrical line), and no energy is dissipated in the failure process. Clearly, this is physically unacceptable.

In order to remedy this well-accounted for fact, reference [55] proposed the use of the so-called "fracture energy regularization technique", nowadays used in many FE applications. This strategy is followed in this work, as it is extremely convenient from the computational standpoint, while guaranteeing the correct conservation of dissipated energy upon mesh refinement.

It has to be remarked that this technique constitutes a crucial and appealing "lost-link" between Continuum and Fracture Mechanics, two disciplines that have often been presented as wide apart ways of approaching Failure Mechanics.

The fracture energy regularization technique is based on the assumption that dissipation takes place in a band only one element thick, irrespective of the element size. The basic concept consists on modifying the softening law in such a way that the energy dissipated over a completely degraded finite element be equal to a given value, which depends on the fracture energy of the material and on the element size. For each element, the material characteristic length ([56], [57]) is approximated by the element characteristic length $l_{\mathrm{ch}}$ [58], which depends on the geometric dimensions of the element and measures the computational width of the fracture zone. The specific dissipated energy $\mathcal{D}$ is then scaled for each element so that the equation

$$
\mathcal{D} l_{\mathrm{ch}}=\mathcal{G}_{I I}
$$

holds, where $\mathcal{G}_{I I}$ is the mode II fracture energy of the material, regarded to be a material property. As it is shown below, this makes the softening modulus $H_{S}$, 
which defines the softening response, dependent on the element size. It also sets a maximum size for the elements that can be used in the analysis. The procedure is as follows:

\subsubsection{Rate independent behaviour}

Consider an ideal experiment in which the load increases monotonically and quasistatically from an initial unstressed state to another in which full degradation takes place.

Using the rate independent version of the damage model, the specific energy dissipated in the process is:

$$
\begin{aligned}
\mathcal{D} & =\int_{t=0}^{t=\infty} \dot{\mathcal{D}} d t \\
& =\int_{t=0}^{t=\infty} W_{\mathrm{dev}}^{e} \dot{d} d t \\
& =\frac{2 / 3}{2(2 G)} \int_{r=r_{0}}^{r=\infty} r^{2} d^{\prime} d r
\end{aligned}
$$

where we have used Eqs. (3.22), (3.18), (3.36), (3.4), (3.8) and the rate of damage can be expressed as $\dot{d}=d^{\prime} \dot{r}$.

We will consider in the following both the cases of linear and exponential softening:

- Linear softening:

Using Eq. (3.9), $d^{\prime}=\left(1+H_{S}\right) r_{0} / r^{2}$, for $r_{0} \leq r \leq r_{u}$, with $r_{u}=$ $r_{0}\left(1+1 / H_{S}\right)$, and $d^{\prime}=0$, otherwise. Recalling that $r_{0}=\sigma_{o}$, integrating and equating $\mathcal{D}=\mathcal{G}_{I I} / l_{\mathrm{ch}}$, we have

$$
\mathcal{D}=\left(1+\frac{1}{H_{S}}\right) \frac{\sigma_{o}^{2}}{2(2 G)}=\frac{\mathcal{G}_{I I}}{l_{\mathrm{ch}}}
$$

and, therefore,

$$
H_{S}=\frac{\bar{H}_{S} l_{\mathrm{ch}}}{1-\bar{H}_{S} l_{\mathrm{ch}}} \quad \geq 0
$$

where $\bar{H}=\sigma_{o}^{2} /\left(3(2 G) \mathcal{G}_{I I}\right)$ depends on the material properties, as $\mathcal{G}_{I I}$ is the mode II fracture energy per unit area, $\sigma_{o}$ is the uniaxial strength and $G$ is the shear modulus. 
- Exponential softening:

Using now Eq. (3.10), $d^{\prime}=\left(r_{0}+2 H_{S} r\right) \exp \left\{-2 H_{S}\left(\frac{r-r_{0}}{r_{0}}\right)\right\} / r^{2}$, for $r_{0} \leq$ $r \leq \infty$. Recalling that $r_{0}=\sigma_{o}$, and integrating, we have

$$
\mathcal{D}=\left(1+\frac{1}{H_{S}}\right) \frac{\sigma_{o}^{2}}{2(2 G)}
$$

which is identical to the result in (3.27).

In the framework of local models and finite element analysis, the state variables are computed at the integration points in terms of the local strain (and/or stress) history. Therefore, the threshold and damage internal variables are defined at the integration points. The characteristic length is thus related to the volume (or area) of each finite element.

For linear simplicial elements, the characteristic length can be taken as the representative size of the element, $l_{\mathrm{ch}}=h_{e}$. In this work, and assuming that the elements are equilateral, the size of the element will be computed as $h_{e}^{2}=$ $(4 / \sqrt{3}) A_{e}$ for triangular elements, $A_{e}$ being the area of the element, and as $h_{e}^{3}=(12 / \sqrt{2}) V_{e}$ for tetrahedral elements, where $V_{e}$ is the volume of the element.

It is clear from Eq. (3.28) that the introduction of the characteristic length implies a limitation on the maximum size of the finite elements used in the mesh, $h_{e} \leq 1 / \bar{H}_{S}$. The greater the elements, the steeper is the softening branch of the response, and, locally, the fracture process is more brittle. For $h_{e}>1 / \bar{H}_{S}$ the ratio $\mathcal{G}_{I I} / h_{e}$ is smaller that the elastic energy $W$ stored by the element, and a dynamic snap-back would occur at the onset of damage.

\subsubsection{Rate dependent behaviour}

Let us now consider an ideal experiment in which the load increases monotonically, and not quasi-statically from an initial unstressed state to another in which full degradation takes place.

Using the rate dependent version of the model, the specific energy dissipated in the process is:

$$
\begin{aligned}
\mathcal{D} & =\int_{t=0}^{t=\infty} \dot{\mathcal{D}} d t \\
& =\int_{t=0}^{t=\infty} W_{\mathrm{dev}}^{e} \dot{d} d t
\end{aligned}
$$




$$
=\frac{2 / 3}{2(2 G)} \int_{r=r_{0}}^{r=\infty}[r+\vartheta \dot{r}]^{2} d^{\prime} d r
$$

where we have used Eqs. (3.22), (3.18), (3.36), and Eq. (3.14) and the rate of damage can be expressed as $\dot{d}=d^{\prime} \dot{r}$.

Comparing Eq. (3.30c) with Eq. (3.26) it is obvious that the first one reduces to the second for a null value of $\vartheta$, or for very slow processes, with $\dot{r}$ tending to zero.

In other cases, the evolution law, Eq. (3.14), ensures that the terms $r$ and $\vartheta \dot{r}$ are of the same order, that is $(\vartheta \dot{r} / r)=\mathcal{O}(1)$. Therefore, it can be assumed that the regularized parameter from Eq. (3.28), that regularizes the rate independent model, will also regularize the rate dependent model, both for linear and exponential softening.

It must be remarked that formats of the regularized damage law different from that of Eq. (3.15) may lead to the necessity of regularizing also the damage retardation time $\vartheta$, making it dependent on the element size ([45], [59]).

\subsection{Update of the damage threshold}

A numerical algorithm needs to be implemented for the time integration of the damage constitutive equations presented in the previous Sections. In the following this algorithm is presented, in a strain-driven form which leads to a completely closed-form algorithm to integrate the stress tensor in time. This is most appropriate within the context of the application of the finite element method.

Each time step begins at time $t_{n}$ with all state variables known and it ends at time $t_{n+1}$ with the state variables updated according to the given total strain tensor $\varepsilon_{n+1}$. The time step size is $\Delta t=t_{n+1}-t_{n}$.

For the rate independent model, Eq. (3.8) allows to compute the current value for $r_{n+1}$ in terms of the current value of $\tau_{n+1}$, which in turn, depends explicitly on the current strains $\boldsymbol{\varepsilon}_{n+1}$ (see Eqs. (3.36) and (3.4)). After this, the damage index $d_{n+1}=d_{n+1}\left(r_{n+1}\right)$ is explicitly computed in terms of the corresponding current value of the damage threshold, using the appropriate expression, Eqs. (3.9) or (3.10).

For the rate dependent model, the only difference is the updating of the damage threshold $r_{n+1}$ when evolution of the damage occurs, that is, upon loading conditions. This may be evaluated using a generalized mid-point rule to integrate 
Eq. (3.14), i.e.,

$$
r_{n+1}=r_{n}+\Delta r_{n+1}=r_{n}+\frac{\Delta t}{\vartheta}\left\langle\tau_{\alpha}-r_{\alpha}\right\rangle
$$

where $\tau_{\alpha}$ and $r_{\alpha}$ are defined by:

$$
\begin{aligned}
\tau_{\alpha} & =(1-\alpha) \tau_{n}+\alpha \tau_{n+1} \\
r_{\alpha} & =(1-\alpha) r_{n}+\alpha r_{n+1}
\end{aligned}
$$

The choice $\alpha=1$ in Eq. (3.31) corresponds to a backward-Euler difference scheme. It is well known that the algorithm of Eq. (3.31) is unconditionally stable for $\alpha \geq 0.5$ and second order accurate only for $\alpha=0.5$ (Crank-Nicholson or trapezoidal rule), which allows the use of larger time step sizes for rate-dependent analysis.

Substituting Eqs. (3.32a) and (3.32b) into Eq. (3.31), yields

$$
r_{n+1}=\max \left\{r_{n}, \frac{1}{1+\alpha \frac{\Delta t}{\vartheta}}\left[\left(1-(1-\alpha) \frac{\Delta t}{\vartheta}\right) r_{n}+\frac{\Delta t}{\vartheta} \tau_{\alpha}\right]\right\}
$$

It is worth to remark that the presented method for the integration of the damage threshold is also valid for the rate independent model, noting that for $\vartheta=0$ and $\alpha=1$, Eq. (3.33) simply reduces to the satisfaction of the monotonicity and consistency conditions

$$
r_{n+1}=\max \left\{r_{n}, \tau_{n+1}\right\}
$$

\subsection{Tangent operator}

\subsubsection{Rate independent damage}

Differentiating Eq.(3.3) with respect to time, we obtain

$$
\dot{\mathbf{s}}=(1-d) \dot{\overline{\mathbf{s}}}-\dot{d} \overline{\mathbf{s}}
$$

The effective deviatoric stresses $\overline{\mathbf{s}}$ can be computed in terms of the total strain tensor $\varepsilon$ as

$$
\overline{\mathbf{s}}=\mathbf{C}_{\mathrm{dev}}: \varepsilon
$$

where $\mathbf{C}_{\mathrm{dev}}=2 G\left[\mathbf{I}-\frac{1}{3}(\mathbf{1} \otimes \mathbf{1})\right]$ is the usual (fourth order) linear-elastic deviatoric constitutive tensor, $\mathbf{I}$ is the (fourth order) unit tensor and (:) denotes the 
tensor product contracted on two indices. Differentiating this with respect to time, we have

$$
\dot{\overline{\mathrm{s}}}=\mathrm{C}_{\mathrm{dev}}: \dot{\varepsilon}
$$

On the other hand, the time derivative of the damage index is

$$
\dot{d}=d^{\prime} \dot{r}
$$

where the derivative $d^{\prime}=d^{\prime}(r)$ can be obtained from Eqs.(3.9)-(3.10). On loading, consistency requires that $\dot{r}=\dot{\tau}$, and therefore, differentiating Eq. (3.4), we can write

$$
\dot{r}=\dot{\tau}=\frac{1}{\tau} \overline{\mathbf{s}}: \dot{\overline{\mathbf{s}}}=\frac{3}{2} \frac{2 G}{\tau} \overline{\mathbf{s}}: \dot{\mathbf{e}}
$$

On unloading, it is $\dot{r}=0$. Substituting this result in Eq. (3.38), and the result in Eq. (3.35), yields the desired expression

$$
\dot{\mathbf{s}}=\mathbf{C}_{\mathrm{dev}}^{\mathrm{tan}}: \dot{\varepsilon}
$$

with

$$
\mathbf{C}_{\mathrm{dev}}^{\tan }=(1-d)(2 G)\left[\mathbf{I}-\frac{1}{3}(\mathbf{1} \otimes \mathbf{1})\right]-h(\overline{\mathbf{s}} \otimes \overline{\mathbf{s}})
$$

where $\mathbf{I}$ is the (fourth order) unit tensor, $\mathbf{1}$ is the (second order) unit tensor and the scalar coefficient $h$ is

$$
h=\left\{\begin{array}{cc}
\frac{3}{2} \frac{2 G}{\tau} d^{\prime} & \text { for loading } \\
0 & \text { for unloading }
\end{array}\right.
$$

The complete constitutive tensor includes the volumetric part:

$$
\mathbf{C}^{\tan }=K(\mathbf{1} \otimes \mathbf{1})+(1-d)(2 G)\left[\mathbf{I}-\frac{1}{3}(\mathbf{1} \otimes \mathbf{1})\right]-h(\overline{\mathbf{s}} \otimes \overline{\mathbf{s}})
$$

which defines the rate constitutive equation

$$
\dot{\boldsymbol{\sigma}}=\mathrm{C}_{\text {tan }}: \dot{\boldsymbol{\varepsilon}}
$$




\subsubsection{Rate dependent damage}

In the case of rate dependent damage the determination of $\dot{r}$ comes from differentiating Eq. (3.31) with respect to time, to yield:

$$
\dot{r}=\frac{\alpha \frac{\Delta t}{\vartheta}}{1+\alpha \frac{\Delta t}{\vartheta}} \dot{\tau}
$$

Comparing Eqs. (3.39) and (3.45), it is obvious that the tangent operator for the rate dependent damage case has the same expression of Eq. (3.41) with the coefficient $h$ given by

$$
h=\left\{\begin{array}{cc}
\frac{\alpha \frac{\Delta t}{\vartheta}}{1+\alpha \frac{\Delta t}{\vartheta}} \frac{3}{2} \frac{2 G}{\tau} d^{\prime} & \text { for loading } \\
0 & \text { for unloading }
\end{array}\right.
$$

Note that for large values of $\Delta t / \vartheta$ the rate independent case is recovered.

\subsection{Stabilization parameter}

The stabilization techniques discussed in the previous section are designed to provide global stability to the incompressible elasto-damage problem. For the J2-damage model proposed above, the definition of the stabilization parameter is straight-forward, as the secant (damaged) shear modulus is simply $G^{*}=(1-d) G$ :

$$
\tau_{e}=\frac{c h_{e}^{2}}{2 G^{*}}=\frac{c h_{e}^{2}}{(1-d) 2 G}
$$




\section{Numerical examples}

The formulation presented in the preceding sections is illustrated below in a number of benchmark problems. Performance of the standard irreductible, the standard mixed and the proposed mixed formulations are tested considering $2 D$ plane-strain 3-noded linear triangular meshes. The examples involve compressible elasticity and isotropic J2-damage with exponential softening.

The following material properties are assumed: Young's modulus $E=10$ $\mathrm{MPa}$, Poisson's ratio $\nu=0.3$ (recall that $G=E / 2(1+\nu), K=E / 3(1-2 \nu)$ ), uniaxial damage stress $\sigma_{o}=10 \mathrm{KPa}$ and mode II fracture energy $G_{I I}=200$ $\mathrm{J} / \mathrm{m}^{2}$. A value $c=1$ is taken for the evaluation of $\tau_{e}$. A value $c^{\prime}=10$ is used for the evaluation of the viscous regularization.

The Newton-Raphson method, combined with a line search procedure is used to solve the non-linear system of equations arising from the spatial and temporal discretization of the weak form of the stabilized problem. In all cases 200 equal time steps are performed to complete the analyses.

Convergence of a step is attained when the ratio between the iterative and the incremental norm of the computed displacements is lower than 0.01 (1\%). Calculations are performed with an enhanced version of the finite element program COMET [60], developed by the authors at the International Center for Numerical Methods in Engineering (CIMNE). Pre and post-processing is done with GiD, also developed at CIMNE [61]. 


\subsection{Singly perforated strip}

The first example is a plane-strain singly perforated strip subjected to axial imposed straining. Because of the double symmetry of the domain and boundary conditions, only one quarter of the domain (the top right quarter) needs to be discretized. Figure 4.1a depicts the original geometry of the problem; dimensions are $20 \times 40 \mathrm{~m} \times \mathrm{m}$ (width $\times$ height) and the radius of the perforation is $r=1$ m. Thickness is $1 \mathrm{~m}$.

The computational domain is divided into an unstructured uniform mesh of 7,336 linear triangles (3,801 nodes) with an average mesh size of $h_{e}=0.25 \mathrm{~m}$, not shown. The pre-processor used tends to introduce patches or equilateral triangles with predominant directions at $-30^{\circ},+30^{\circ}$ and $+90^{\circ}$ with the horizontal axis.

Figure 4.2 shows the results obtained using the standard irreductible formulation, once the shear bands are fully developed and the collapse mechanism can be appreciated ((half)-imposed vertical displacement $\delta=0.10 \mathrm{~m})$. It can be observed that, as expected, the pressure contours present wild spurious oscillations, particularly in the vicinity of the $X$-shaped shear bands. This volumetric locking precludes the formation of really sharp slip lines and this is evident in the plots showing the contours of equivalent deviatoric strain, $\bar{e}=\sqrt{2 / 3}\|\mathbf{e}\|$, and damage index, $d$. Lack of objectivity with respect to the mesh directional bias is evident in the vicinity of the outer lateral boundaries.
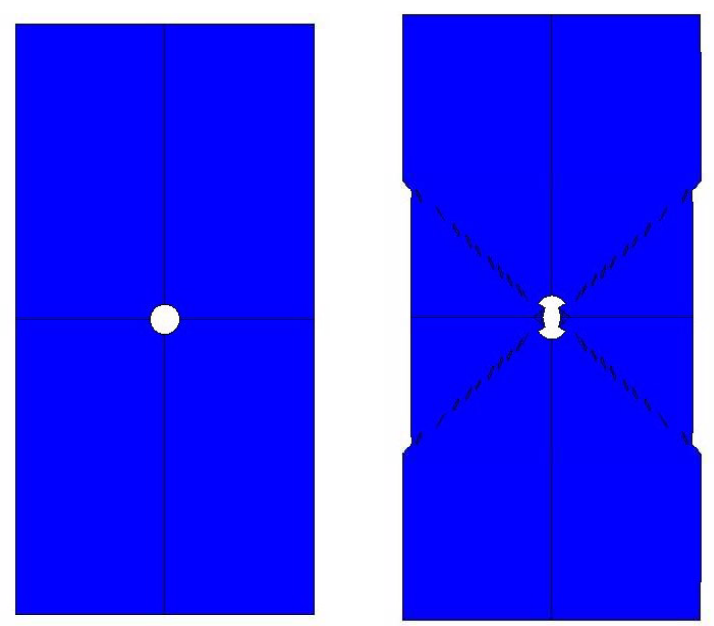

Fig. 4.1: Original and deformed (x 5) geometries for singly perforated strip 


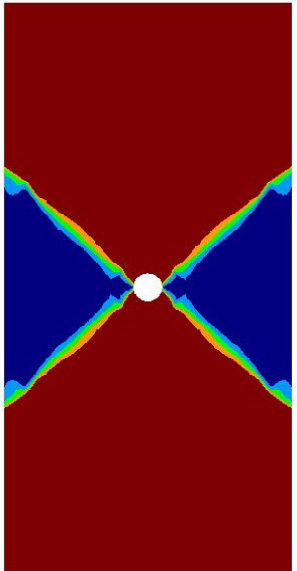

(a)

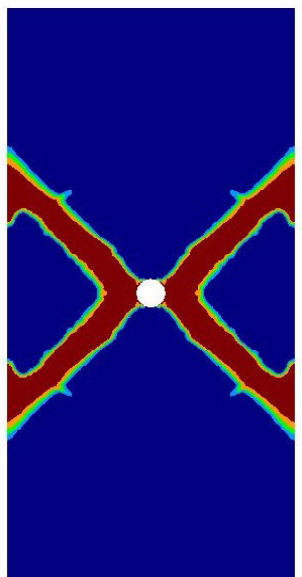

(c)

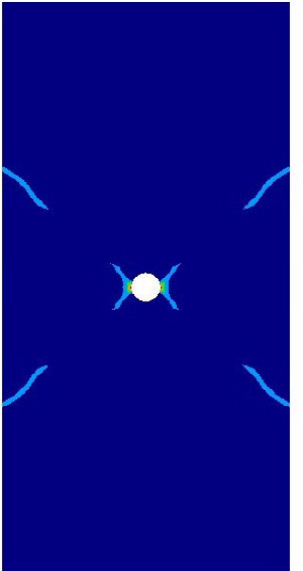

(b)

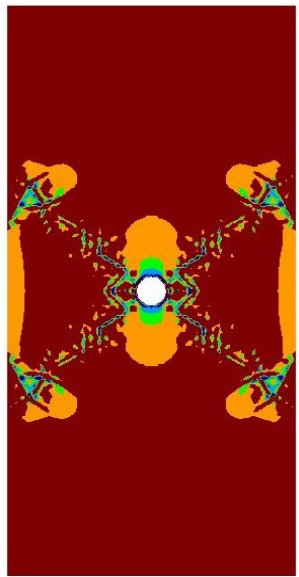

(d)

Fig. 4.2: Results for singly perforated strip using the standard irreductible formulation. Contours for: (a) vertical displacement, (b) equivalent deviatoric strain, (c) damage index and (d) pressure

Figure 4.3 shows the results obtained using the standard mixed $\mathbf{u} / p$ formulation, also for a (half)imposed vertical displacement $\delta=0.10 \mathrm{~m}$. The improvement with respect to the irreductible formulation is spectacular, but the lack of stability is evident in the observed spurious oscillation of the pressure. The resolution of the shear bands is fairly sharp, as shown by the displacement and equivalent deviatoric strain plots. These shear bands form a correct angle of approximately $\pm 45^{\circ}$ with the horizontal axis, but a certain lack of objectivity with respect to the 


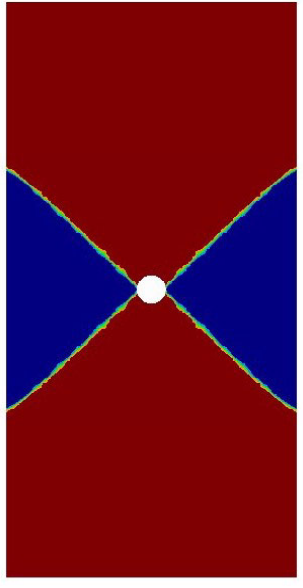

(a)

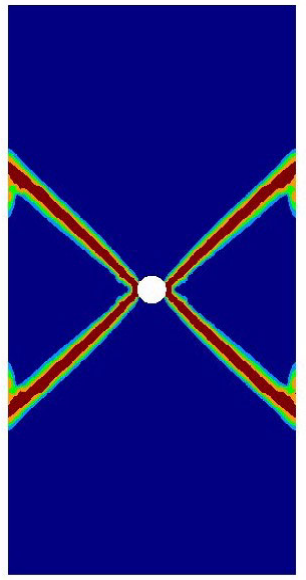

(c)

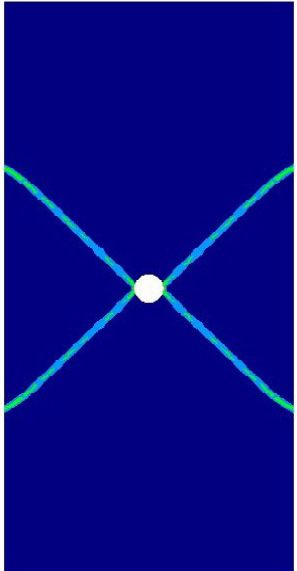

(b)

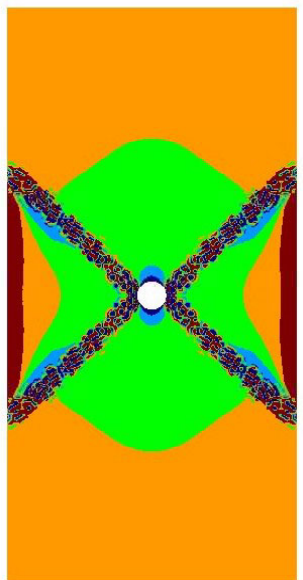

(d)

Fig. 4.3: Results for singly perforated strip with standard mixed formulation. Contours for: (a) vertical displacement, (b) equivalent deviatoric strain, (c) damage index and (d) pressure

mesh directional bias is still evident in the vicinity of the outer lateral boundaries.

Figure 4.4 shows the results obtained using the proposed stabilized mixed $\mathbf{u} / p$ formulation, also for a (half)imposed vertical displacement $\delta=0.10 \mathrm{~m}$. Control on the pressure is completely attained, and no spurious oscillations are observed anywhere in the domain. The resolution of the shear bands is optimal for the mesh used, as shown by the displacement and equivalent deviatoric strain plots. 


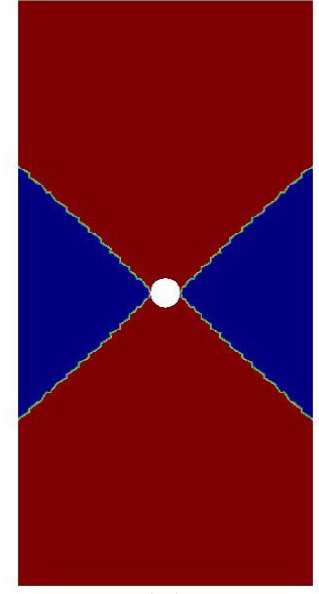

(a)

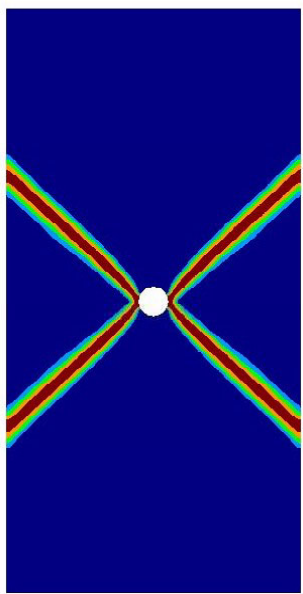

(c)

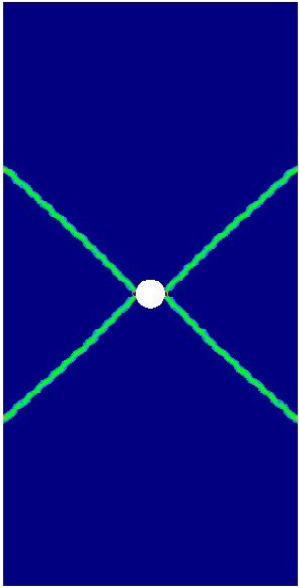

(b)

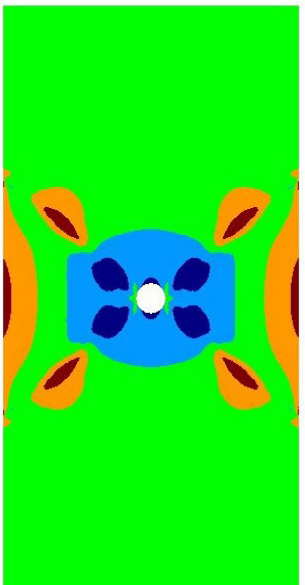

(d)

Fig. 4.4: Results for singly perforated strip with OSGS formulation. Contours for: (a) vertical displacement, (b) equivalent deviatoric strain, (c) damage index and (d) pressure

Discontinuity of the displacement tangential to the slip line occurs across one single element. No indication of "overshoots" or "undershoots" of any magnitude is observed at either side of the discontinuity lines. The deformed shape of the strip (with an amplification factor of 5) is shown in Figure 4.1.

Figure 4.5 shows the evolution of the damage index at four different stages of the analysis, for: (a) $\delta=0.010 \mathrm{~m}$, (b) $\delta=0.015 \mathrm{~m}$, (c) $\delta=0.020 \mathrm{~m}$ and (d) 


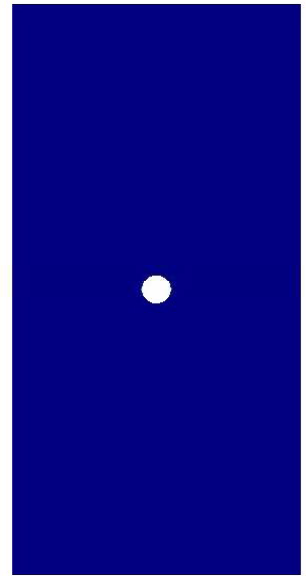

(a)

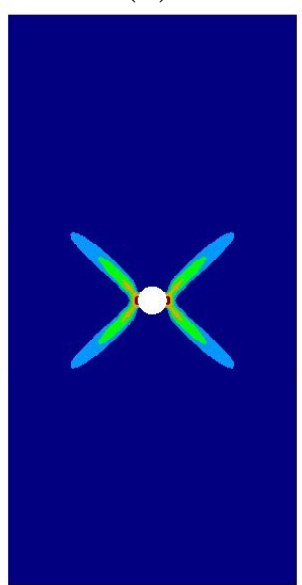

(c)

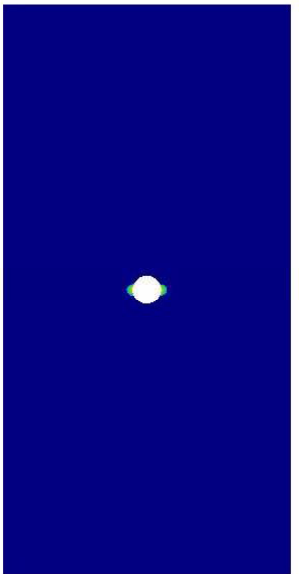

(b)

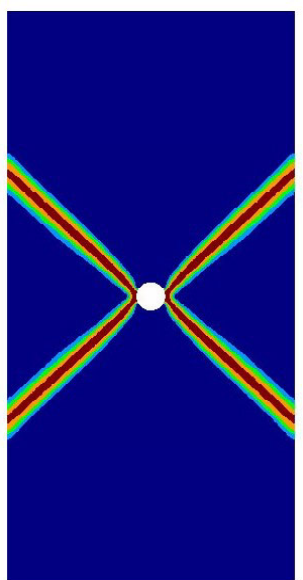

(d)

Fig. 4.5: Evolution of damage for singly perforated strip. Contours for (half)-imposed vertical displacement equal to: (a) 0.010, (b) 0.015, (c) 0.020 and (d) 0.025

$\delta=0.025 \mathrm{~m}$. Note that the localization bands form completely at a very early stage of the analysis, and in a quite "explosive" fashion. The damage index of the elements inside the localization bands reaches values very close to unity, complete shear degradation, very early during the analysis.

Figure 4.6 shows (half)-load vs (half)-imposed vertical displacement curves (recall $1 \mathrm{~m}$ thickness is assumed) obtained with the three formulations: (a) stan- 


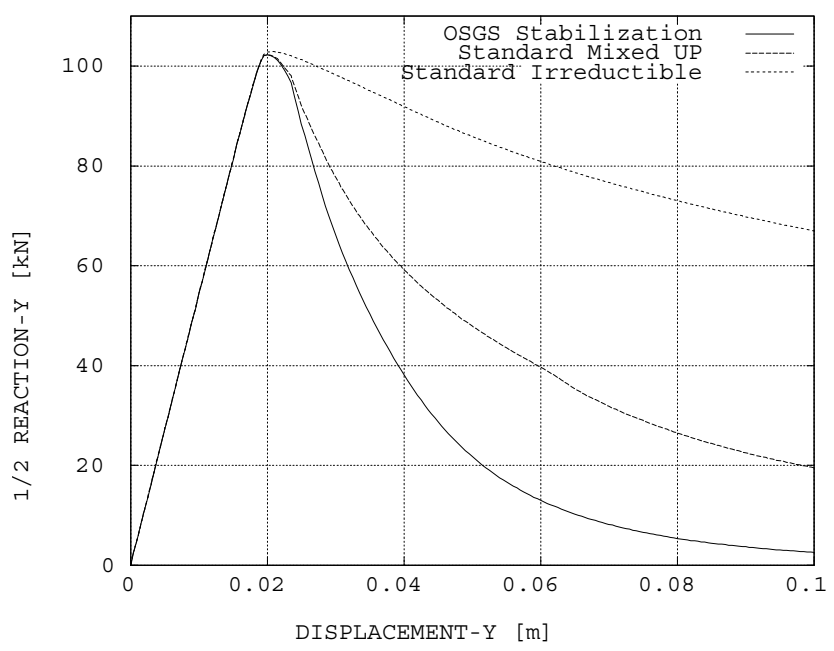

Fig. 4.6: Load versus displacement for simply perforated strip. Comparison among different formulations

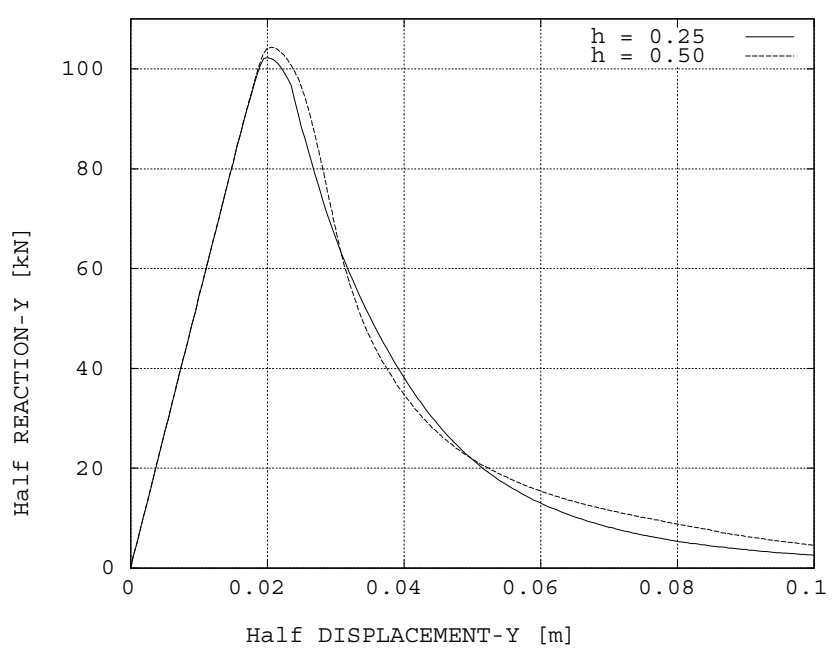

Fig. 4.7: Load versus displacement for simply perforated strip. Comparison between different mesh sizes 
dard irreductible, (b) standard mixed and (c) mixed with OSGS stabilization. Two remarks are in order. First, both the mixed formulations capture reasonably well both the limit load and the general softening trend of the curve, while the irreductible formulation fails to do so almost completely. Second, the effect of the stabilization techniques adopted is obvious in the global softening response, with clear advantage for the proposed OSGS method. As it has been explained, the differences in the responses are due to the different degrees of success of the formulations in removing the volumetric locking induced by the isochoric deformational behaviour inside the shear bands.

Finally, Figure 4.7 shows (half)-load vs (half)-imposed vertical displacement curves ( $1 \mathrm{~m}$ thickness is assumed) obtained with two uniform unstructured meshes with two element sizes: (a) $h_{e}=0.25 \mathrm{~m}$ and (b) $h_{e}=0.50 \mathrm{~m}$ (1,820 elements, 977 nodes). Note that the overall global response is satisfactorily similar upon mesh refinement, with the total area under the load-displacement curve converging to the correct amount of mechanical dissipation dissipated to create the localization bands. No spurious brittleness is observed when the size of the elements is reduced.

\subsection{Doubly perforated strip}

The second example is a plane-strain doubly perforated strip subjected to axial imposed straining. Because of the double symmetry of the domain and boundary conditions, only one quarter of the domain (the top right quarter) is discretized. Figure 4.8a depicts the original geometry of the problem; dimensions are $20 \times 40$ $\mathrm{m} \times \mathrm{m}$ (width $\times$ height) and the radius of the perforations is $r=1 \mathrm{~m}$. Thickness is $1 \mathrm{~m}$.

This example is interesting because, as it will be shown below, the symmetric collapse mechanism consists of multiple shear bands that intersect each other. Therefore, it is an adequate test to assess the ability of the different formulations to deal with such a complex situation.

The computational domain is divided into an unstructured uniform mesh of 7,289 linear triangles (3,780 nodes) with an average mesh size of $h_{e}=0.25 \mathrm{~m}$. 
Figure 4.9 shows the results obtained using the standard irreductible formulation, once the shear bands are fully developed and the collapse mechanism can be appreciated ((half)imposed vertical displacement $\delta=0.10 \mathrm{~m})$. As expected, the pressure contours present wild spurious oscillations, particularly in the vicinity of the double $X$-shaped shear bands. This volumetric locking precludes the formation of really sharp slip lines and this is evident in the plots showing the contours of equivalent deviatoric strain and damage index. Lack of objectivity with respect to the mesh directional bias is evident, as the shear bands form an incorrect angle of $\pm 30^{\circ}$ with the horizontal axis.

Figure 4.10 shows the results obtained using the standard mixed $\mathbf{u} / p$ formulation, also for a (half)-imposed vertical displacement $\delta=0.10 \mathrm{~m}$. The improvement with respect to the irreductible formulation is remarkable, as the shear bands form a correct angle of approximately $\pm 45^{\circ}$ with the horizontal axis, but a certain lack of objectivity with respect to the mesh directional bias is evident in the vicinity of the outer lateral boundaries, where some of the slip lines bifurcate. The lack of stability of the formulation is evident in the observed spurious oscillation of the pressure. The resolution of the shear bands is fairly sharp, as shown by the displacement and equivalent deviatoric strain plots.

Figure 4.11 shows the results obtained using the proposed stabilized mixed $\mathbf{u} / p$ formulation, also for a (half)-imposed vertical displacement of $\delta=0.10 \mathrm{~m}$. Full control on the pressure is attained, and no spurious oscillations are observed
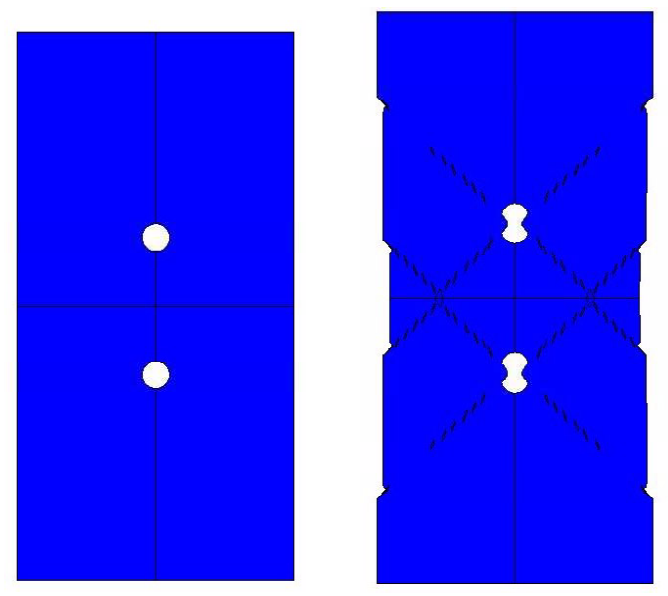

Fig. 4.8: Original and deformed (x 5) geometries for doubly perforated strip 


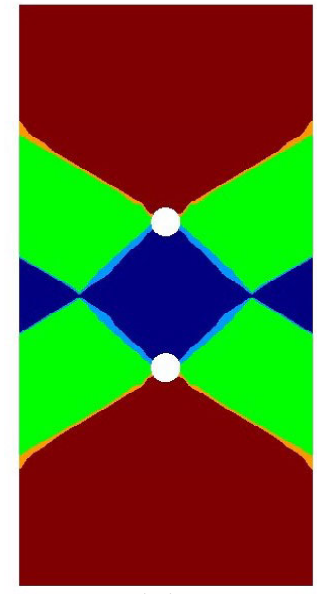

(a)

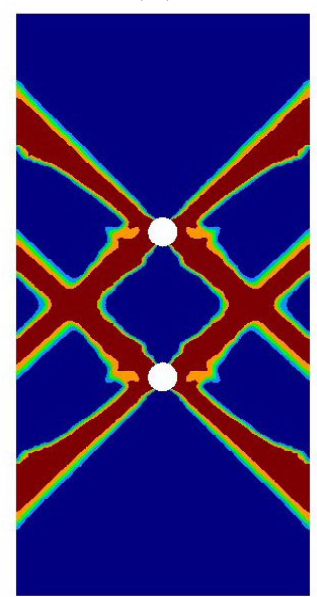

(c)

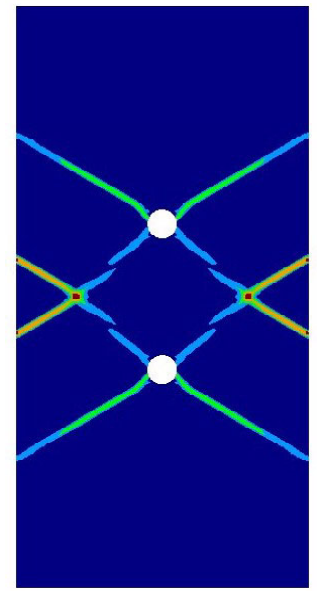

(b)

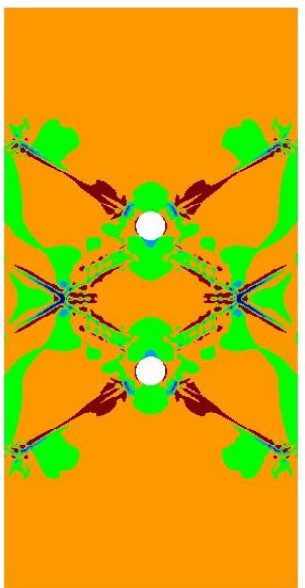

(d)

Fig. 4.9: Results for doubly perforated strip using the standard irreductible formulation. Contours for: (a) vertical displacement, (b) equivalent deviatoric strain, (c) damage index and (d) pressure

anywhere. The resolution of the shear bands is optimal, as shown by the displacement and the equivalent deviatoric strain contour plots, where the discontinuities occur across one single element. No spurious branching of the slip lines occurs near the lateral boundaries. The deformed shape of the perforated strip (with a displacement amplification factor of 5 ) is shown in Figure 4.8b.

Figure 4.12 shows the evolution of the damage index at four different stages 


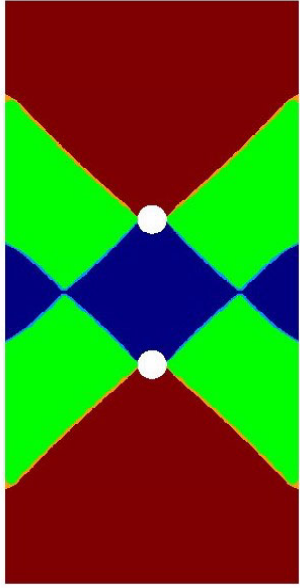

(a)

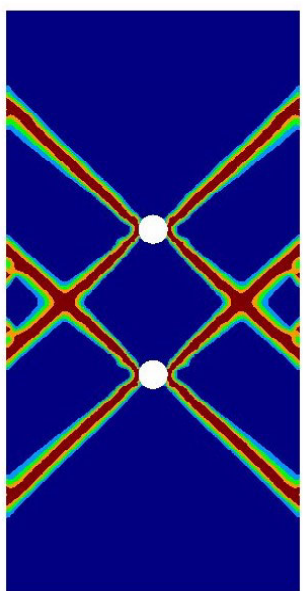

(c)

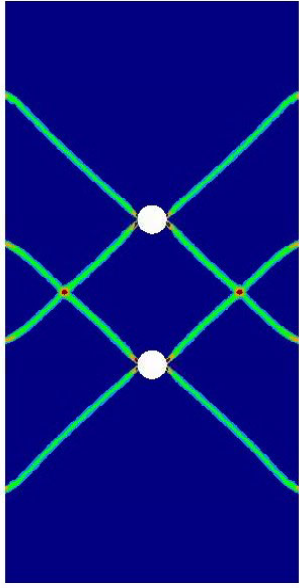

(b)

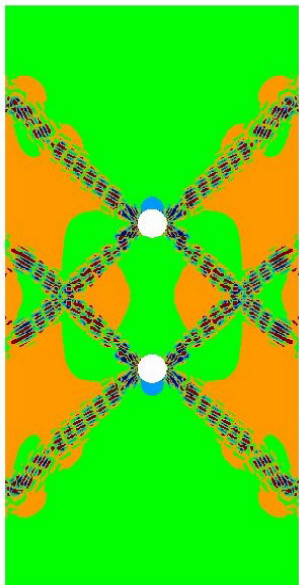

(d)

Fig. 4.10: Results for doubly perforated strip with standard mixed formulation. Contours for: (a) vertical displacement, (b) equivalent deviatoric strain, (c) damage index and (d) pressure

of the analysis, for: (a) $\delta=0.010 \mathrm{~m}$, (b) $\delta=0.020 \mathrm{~m}$, (c) $\delta=0.030 \mathrm{~m}$ and (d) $\delta=0.040 \mathrm{~m}$. Also in this example the localization bands form completely at a very early stage of the analysis, with elements almost completely damaged inside the localization bands.

Figure 4.13 shows (half)-load vs (half)-imposed vertical displacement curves (recall $1 \mathrm{~m}$ thickness is assumed) obtained with the three formulations: (a) stan- 


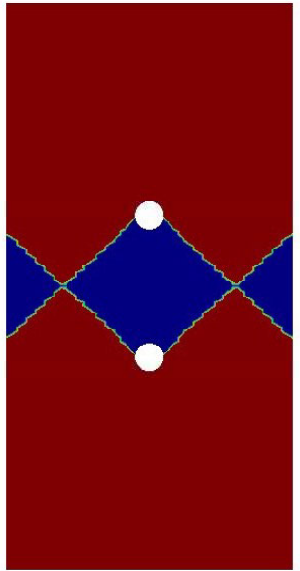

(a)

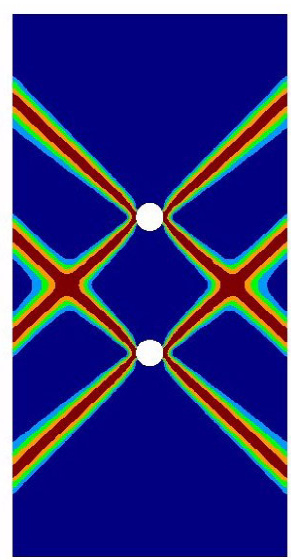

(c)

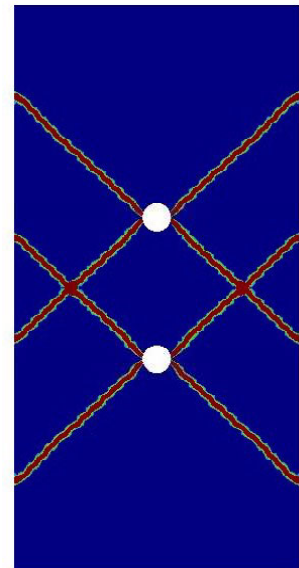

(b)

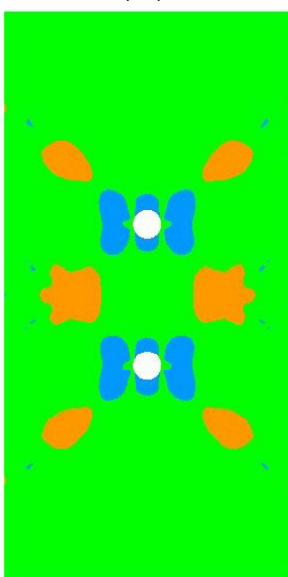

(d)

Fig. 4.11: Results for doubly perforated strip with OSGS formulation. Contours for: (a) vertical displacement, (b) equivalent deviatoric strain, (c) damage index and (d) pressure

dard irreductible, (b) standard mixed and (c) mixed with OSGS stabilization. The remarks referred to the previous example also apply here. Note that the energy dissipated in this example (the area below the curve in the plot) is approximately double than the one dissipated in the previous example, see Figure 4.7. This is because the number, and surface, of the discontinuities created is approximately twice as much.

Finally, Figure 4.14 shows (half)-load vs (half)-imposed vertical displacement 


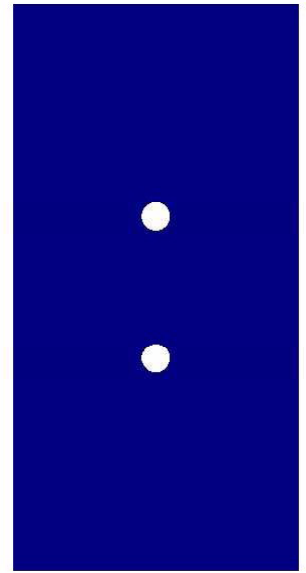

(a)

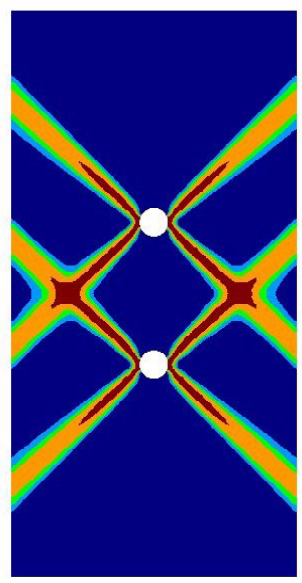

(c)

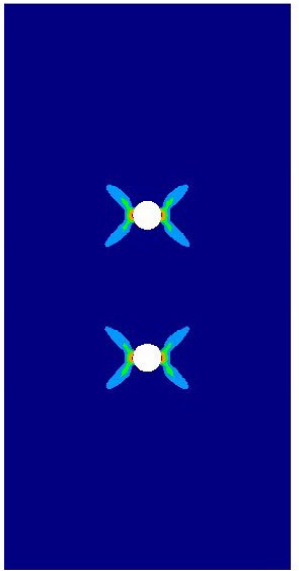

(b)

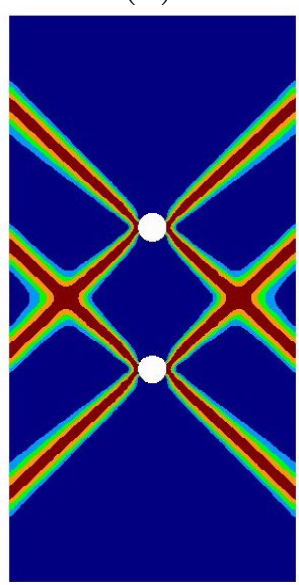

$(d)$

Fig. 4.12: Evolution of damage for doubly perforated strip. Contours for (half)-imposed vertical displacement equal to: (a) 0.010, (b) 0.020, (c) 0.030 and (d) 0.040

curves ( $1 \mathrm{~m}$ thickness is assumed) obtained with two uniform unstructured meshes with two element sizes: (a) $h_{e}=0.25 \mathrm{~m}$ and (b) $h_{e}=0.50 \mathrm{~m}$ (1,794 elements, 965 nodes). No spurious brittleness is observed when the size of the elements is reduced. 


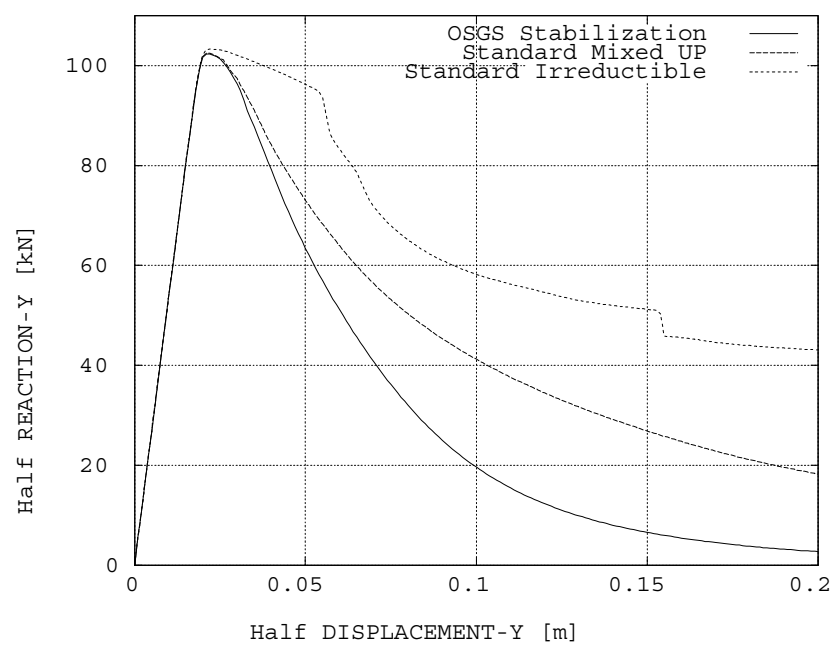

Fig. 4.13: Load versus displacement for doubly perforated strip. Comparison among different formulations

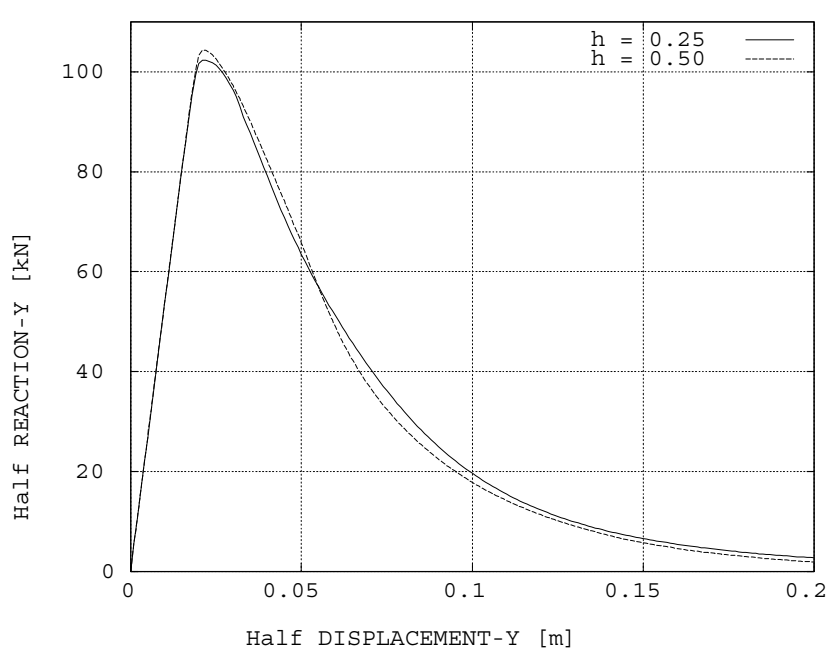

Fig. 4.14: Load versus displacement for doubly perforated strip. Comparison between different mesh sizes 


\subsection{Multiply perforated strip}

The last example is a plane-strain strip with four circular perforations subjected to axial imposed straining. Because of the double symmetry of the domain and boundary conditions, only one quarter of the domain (the top right quarter) is discretized. Figure 4.15a depicts the original geometry of the problem; as in the previous examples, dimensions are $20 \times 40 \mathrm{~m} \times \mathrm{m}$ (width $\times$ height) and the radius of the perforations is $r=1 \mathrm{~m}$. Thickness is $1 \mathrm{~m}$.

The interest of this last example is that in it, as it will be shown below, the symmetric collapse mechanism consists of multiple shear bands that intersect each other and connect the perforations among them. Therefore, it is an adequate test to assess the ability of the different formulations to deal with such a complex situation.

The computational domain is divided into an unstructured uniform mesh of 7,310 linear triangles (3,810 nodes) with an average mesh size of $h_{e}=0.25 \mathrm{~m}$.

Figure 4.16 shows the results obtained using the standard irreductible formulation, once the shear bands are fully developed and the collapse mechanism can be appreciated ((half)-imposed vertical displacement $\delta=0.10 \mathrm{~m})$. The pressure contours present severe oscillations, particularly in the vicinity of the $X$-shaped shear bands. Volumetric locking is evident in the plots showing the contours of equivalent deviatoric strain and damage index. Lack of objectivity with respect
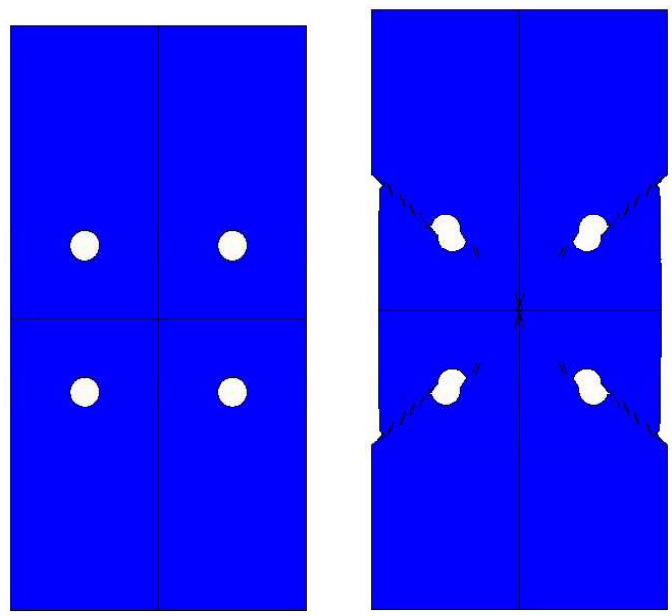

Fig. 4.15: Original and deformed (x 5) geometries for multiply perforated strip 


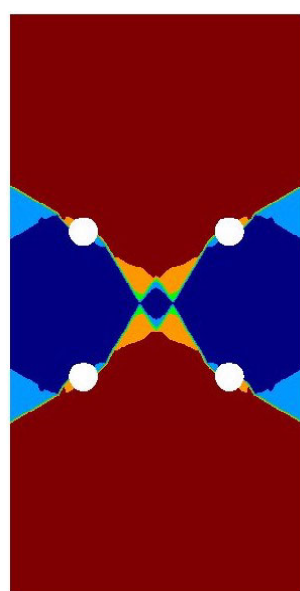

(a)

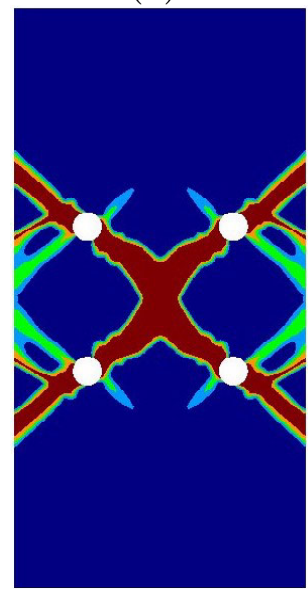

(c)

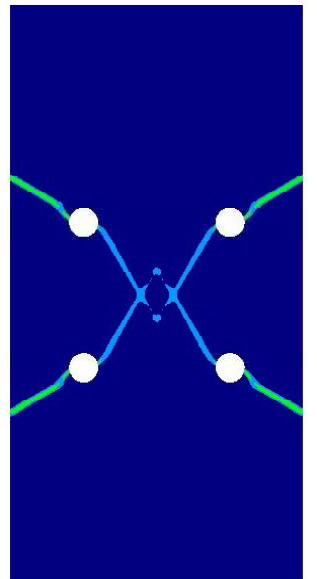

(b)

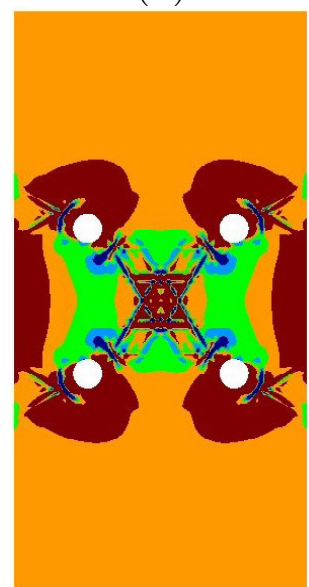

(d)

Fig. 4.16: Results for multiply perforated strip using the standard irreductible formulation. Contours for: (a) vertical displacement, (b) equivalent deviatoric strain, (c) damage index and (d) pressure

to the mesh directional bias is clear, as the outer shear bands bend to form an incorrect angle of $\pm 30^{\circ}$ with the horizontal axis. The inner shear bands also show an unrealistic mesh bias, as they fail to connect at the centre of the specimen. The outer shear bands show a pronounced kink, following the mesh preference alignment, and they branch spuriously near the lateral outer boundaries.

Figure 4.17 shows the results obtained using the standard mixed $\mathbf{u} / p$ formulation, for the same (half)-vertical displacement. In this case, the shear bands form 


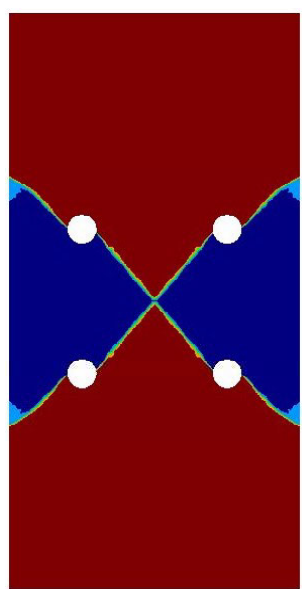

(a)

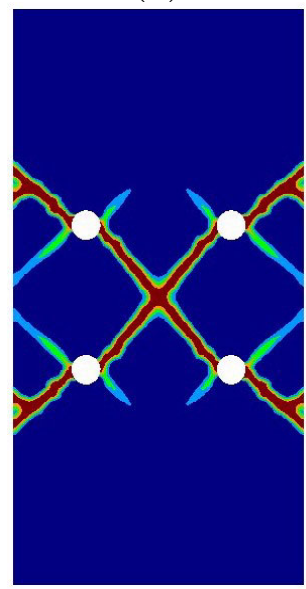

(c)

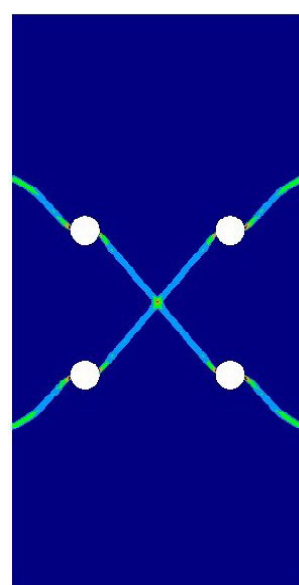

(b)

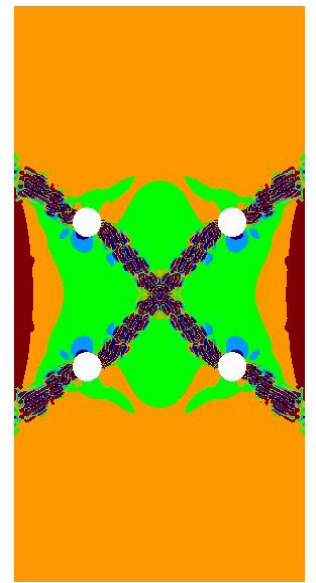

(d)

Fig. 4.1\%: Results for multiply perforated strip with standard mixed formulation.

Contours for: (a) vertical displacement, (b) equivalent deviatoric strain, (c) damage index and (d) pressure

a correct angle of approximately $\pm 45^{\circ}$ with the horizontal axis, although they show mesh dependent kinks and branches as they approach the vertical lateral boundaries. The inner bands form correctly, crossing each other at the centre of the specimen. The lack of local stability of the formulation also shows in the severe oscillations of the pressure inside and in the vicinity of the shear bands. 


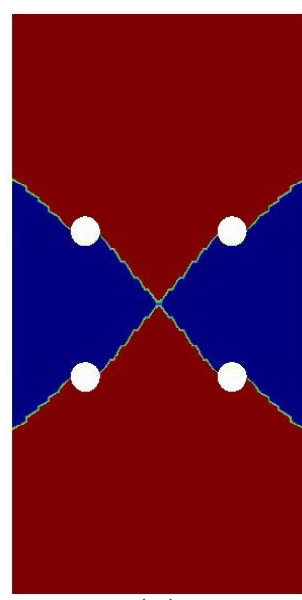

(a)

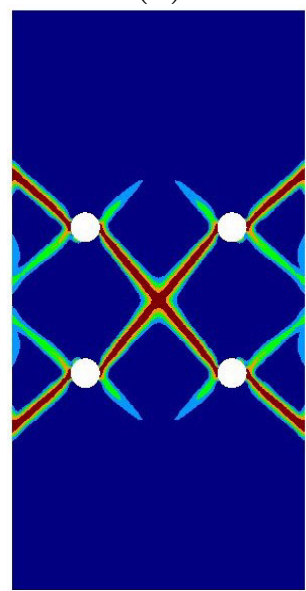

(c)

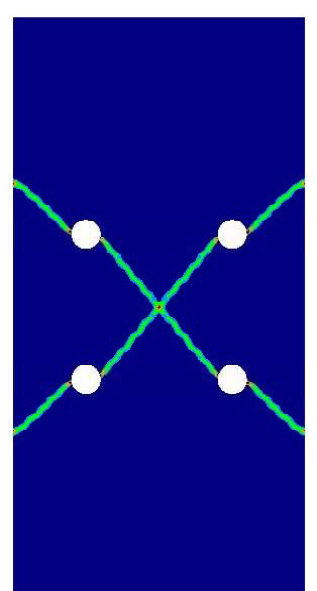

(b)

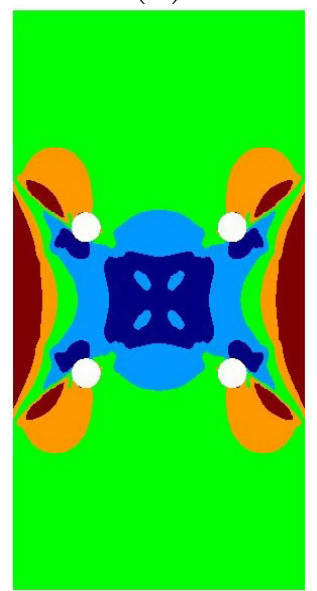

(d)

Fig. 4.18: Results for multiply perforated strip with OSGS formulation.

Contours for: (a) vertical displacement, (b) equivalent deviatoric strain, (c) damage index and (d) pressure

Figure 4.18 shows the results obtained using the proposed stabilized mixed $\mathbf{u} / p$ formulation, also for a (half)-imposed vertical displacement $\delta=0.10 \mathrm{~m}$. The results obtained with this method are optimal, and no spurious oscillations are observed in the pressure contours. The resolution of the shear bands is optimal, as shown by the displacement and equivalent deviatoric strain plots, with the discontinuities occurring across one single element. No spurious kinking or branching occurs near the lateral boundaries. The deformed shape of the strip 


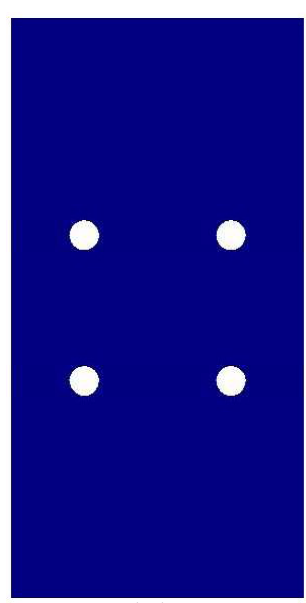

(a)

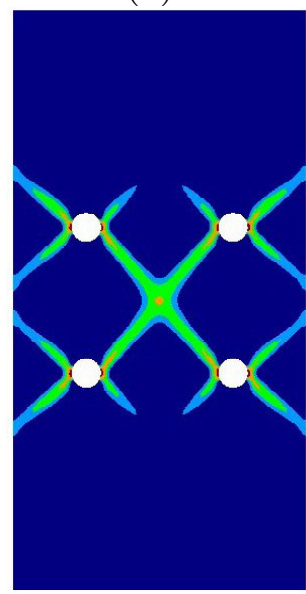

(c)

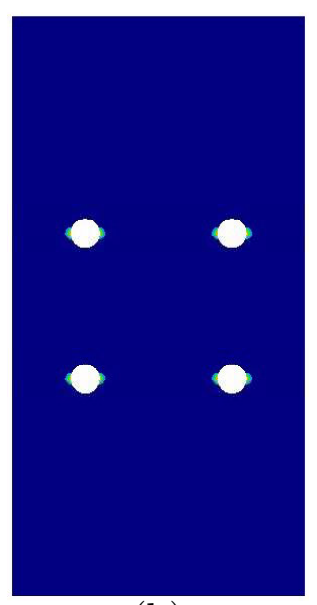

(b)

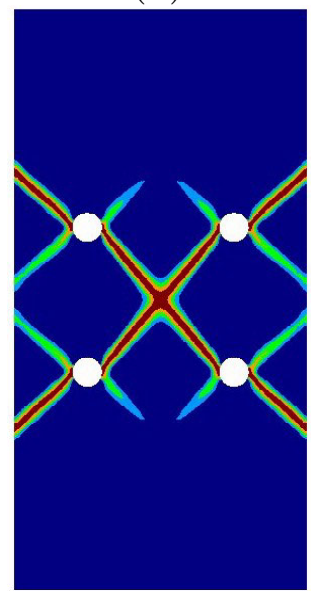

(d)

Fig. 4.19: Evolution of damage for multiply perforated strip. Contours for (half)-imposed vertical displacement equal to: (a) 0.010, (b) 0.015, (c) 0.020 and (d) 0.025

(with an amplification factor of 5) is shown in Figure 4.15b.

Figure 4.19 shows the evolution of the damage index at four different stages of the analysis, for: (a) $\delta=0.010 \mathrm{~m}$, (b) $\delta=0.015 \mathrm{~m}$, (c) $\delta=0.020 \mathrm{~m}$ and (d) $\delta=0.025 \mathrm{~m}$.

Figure 4.20 shows (half)-load vs (half)-imposed vertical displacement curves (recall $1 \mathrm{~m}$ thickness is assumed) obtained with the three formulations: (a) standard irreductible, (b) standard mixed and (c) mixed with OSGS stabilization. 


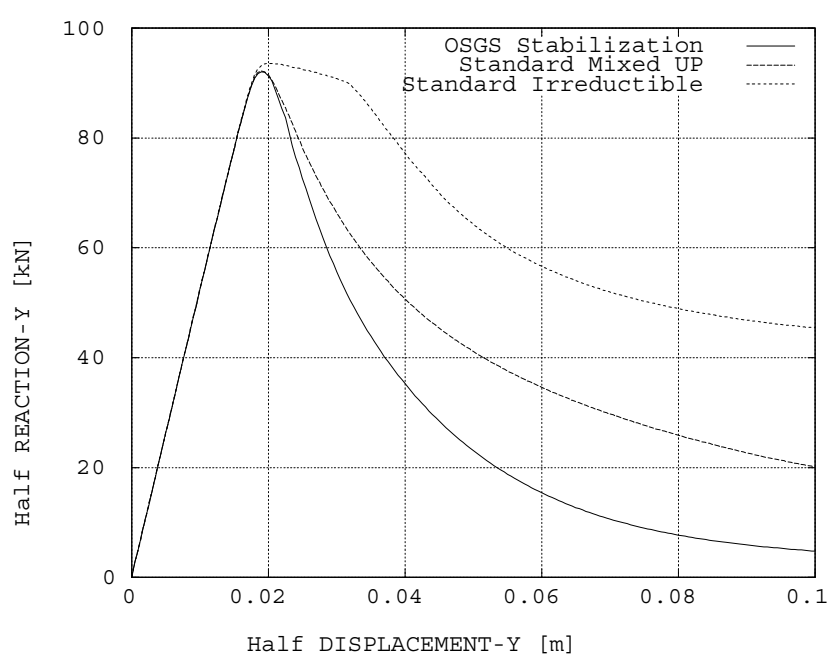

Fig. 4.20: Load versus displacement for multiply perforated strip. Comparison among different formulations

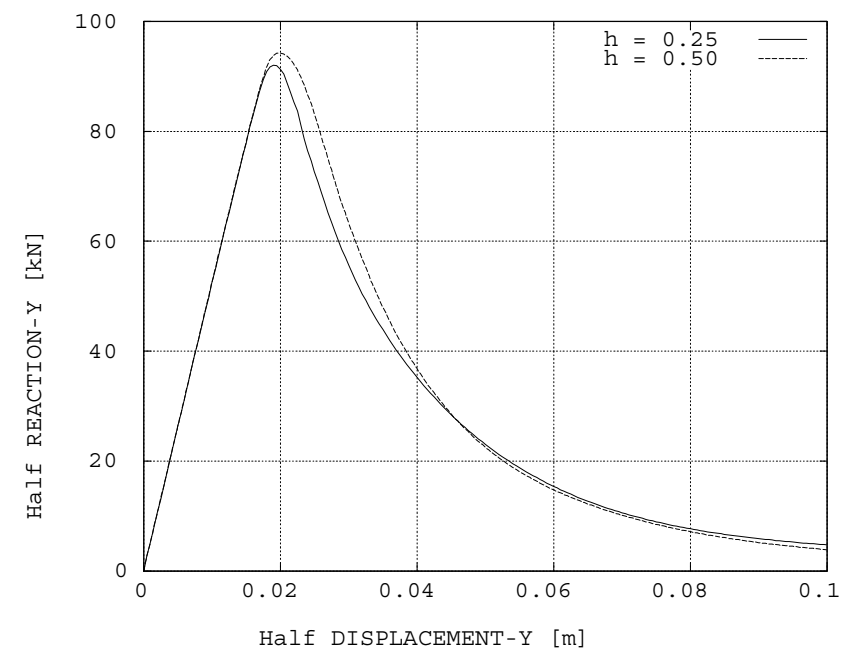

Fig. 4.21: Load versus displacement for multiply perforated strip. Comparison between different mesh sizes 
The remarks referred to the previous examples also apply here.

Finally, Figure 4.21 shows (half)-load vs (half)-imposed vertical displacement curves (1m thickness is assumed) obtained with two uniform unstructured meshes with two element sizes: (a) $h_{e}=0.25 \mathrm{~m}$ and (b) $h_{e}=0.50 \mathrm{~m}$ (1,810 elements, 977 nodes). Once again, no spurious brittleness is observed when the size of the elements is reduced. 


\section{Conclusions}

This paper shows the application of stabilized mixed linear simplex (triangles and tetrahedra) to the solution of shear band localization problems via a local isotropic continuum J2 damage model.

A fully stable formulation of the problem is obtained using the orthogonal sub-grid scales $(O S G S)$ approach, which allows equal order interpolation of the displacement and pressure fields. This translates in the achievement of three crucial goals:

(a) the solution of the corresponding localization boundary value problem exists and it is unique,

(b) the position and orientation of the localization bands is independent of the directional bias of the finite element mesh, and

(b) the global post-peak load-deflection curves are independent of the size of the elements in the localization band.

The accomplishment of these fundamental objectives is attained by ensuring both global and local stability of the problem.

A consistent residual viscous regularization is proposed and successfully applied, in order to overcome the convergence difficulties often encountered in applications involving softening behaviour and strain localization. The derived method yields a robust scheme, suitable for engineering applications in $2 \mathrm{D}$ and 3D.

The proposed formulation is shown to attain total control on the pressure field, removing global and local oscillations induced by the incompressibility constraints induced by the material model.

Numerical examples show, on one hand, the tremendous advantage of the mixed formulation over the irreductible one to predict correct failure mechanisms with localized patterns of shear deformation, virtually free from any dependence of 
the mesh directional bias; on the other, stabilization techniques are shown to fully avoid the volumetric locking exhibited by the standard non-stable formulations, yielding a correct global response in the softening regime.

\section{Acknowledgments}

The authors gratefully acknowledge the invaluable help of our colleagues Prof. R. Codina and Prof. S. Oller, expressed in the form of unfailing suggestions and discussions. 


\section{Bibliography}

[1] Aifantis, E.C. (1984). On the microstructural origin of certain inelastic models. Transactions ASME Journal of Engineering Materials Technology, 106, 326-330.

[2] Schereyer, H. and Chen, Z. (1986). One dimensional softening with localization. Journal of Applied Mechanics, ASME, 53, 791-797

[3] Vardoulakis, I. and Aifantis, E.C. (1991). A gradient flow theory of plasticity for granular materials. Acta Mechanica, 87, 197-217.

[4] de Borst, R. and Mulhaus, H.B. (1992). Gradient-dependent plasticity: formulation and algorithm aspect. Int. J.. Num. Meths. in Engng., 35, 521-539.

[5] Pamin, J. (1994). Gradient-Dependent Plasticity in Numerical Simulation of Localization Phenomena. Ph. D. Thesis, TU delft, The Netherlans.

[6] Peerlings, R.H.J., de Borst, R., Brekelmans, W.A. M. and Geers, M.G.D. (1998). Gradient-enhanced damage modelling of concrete failures. Mechanics of Cohesive-Frictional Materials, 4, 339-359.

[7] Jirásek, M. (1998). Nonlocal models for damage and fracture: comparison of approaches. Int. J. Solids and Structures, 35, 4133-4145.

[8] de Borst, R. (2001). Some recent issues in computational failure mechanics. Int. J. Num. Meths. in Eng., 52, 63-95.

[9] de Borst, R. (1991). Simulation of strain localization: a reppraisal of the Cosserat continuum. engineering computations, 8, 317-322. 
[10] Steinmann, P. and Willam, K. (1992). Localization witin the framework of micropolar elastoplasticy. In Advances in Continuum Mechanics. ed. V. Mannl et al. pp. 296-313. Springer Verlag, Berlin.

[11] Needelman, A., (1987). Material rate dependence and mesh sensitivity in localization problems. Comp. Meth. in Appl. Mech. and Eng., 67, 68-75.

[12] Simo, J.C., Oliver, J. and Armero, F. (1993). An analysis of strong discontinuities induced by strain-softening in rate-independent inelastic solids. Computational Mechanics, 12, 49-61.

[13] Oliver, J. (1995). Continuum modeling of strong discontinuities in solid mechanics using damage models. Computational Mechanics, 17, 277-296.

[14] Oliver, J., Cervera, M. and Manzoli, O. (1999). Strong discontinuities and continuum plasticity models: the strong discontinuity approach. Int. J. of Plasticity, 15, 319-351.

[15] Oliver, J., Huespe, A.E. and Samaniego, E. (2003). A study on finite elements for capturing strong discontinuities. Int. for Num. Meths. in Eng., in press.

[16] Chaves, E.W.V. (2003). A Three Dimensional Setting for Strong Discontinuities Modelling in Failure Mechanics. Ph. D. Thesis. University of Catalonia.

[17] de Borst, R. (2002). Fracture in quasi-brittle materials: a review of continuum damage-based approaches. Engineering Fracture Mechanics, 69, 95-112.

[18] Zienkiewicz, O.C. and Taylor, R.L. (2000). The Finite Element Method, Butterworth-Heinemann, Oxford.

[19] Zienkiewicz, O.C., Pastor, M. and Huang, M. (1995). Softening, localization and adaptive remeshing: capture of discontinuous solutions. Comp. Mech., 17, 98-106.

[20] Zienkiewicz O.C., Huang, M. and Pastor, M. (1995). Localization problems in plasticity using finite elements with adaptive remeshing. Int. J. Num. Methods in Geomechanics, 19, 127-148. 
[21] Bonet, J. and Burton, A.J. (1998). A simple average nodal pressure tetrahedral element for incompressible and nearly incompressible dynamic explicit applications. Comm. Num. Meths. in Eng., 14, 437-449.

[22] Zienkiewicz, O.C., Rojek, J., Taylor, R.L. and Pastor, M. (1998). Triangles and tetrahedra in explicit dynamic codes for solids, Int. J. for Num. Meths. in Eng., 43, 565-583.

[23] Klaas, O., Maniatty, A. and Shephard, M.S. (1999). A stabilized mixed finite element method for finite elasticity. Formulation for linear displacement and pressure interpolation, Comp. Meth. in Appl. Mech. and Eng., 180, 65-79.

[24] Taylor, R.L. (1999). A mixed formulation for triangular and tetrahedral elements, In Abascal, R., Domínguez, J. and Bugeda, G., editors, Conference Proceedings on Métodos Numéricos en Ingeniería, SEMNI, Barcelona, Spain.

[25] Dohrmann, C.R., Heinstein, M.W., Jung, J., Key, S.W. and Witkowsky, W.R. (2000). Node-based uniform strain elements for three-node triangular and four-node tetrahedral meshes. Int. Jour. for Num. Meths. in Eng. 47, 1549-1568.

[26] Taylor, R.L. (2000). A mixed-enhanced formulation for tetrahedral elements. Int. Jour. for Num. Meths. in Eng. 47, 205-227.

[27] Bonet, J., Marriot, H. and Hassan, O. (2001). An averaged nodal deformation gradient linear tetrahedral element for large strain explicit dynamic applications. Comm. Num. Meths. in Eng., 17, 551-561.

[28] Bonet, J., Marriot, H. and Hassan, O. (2001). Stability and comparison of different linear tetrahedral formulations for nearly incompressible explicit dynamic applications. Int. Jour. for Num. Meths. in Eng., 50, 119-133.

[29] Oñate, E., Rojek, J., Taylor, R.L. and Zienkiewicz, O.C. (2001) Linear triangles and tetrahedra for incompressible problem usung a finite calculus formulation, Proceedings of European Conference on Computational Mechanics, ECCM.

[30] de Souza Neto, E.A., Pires, F.M.A. and Owen D.R.J. (2003). A new F-barmethod for linear triangles and tetrahedra in the finite strain analysis of 
nearly incompressible solids. Proceedings of VII International Conference on Computational Plasticity, COMPLAS.

[31] Hughes, T.J.R. (1995). Multiscale phenomena: Green's function, Dirichletto Neumann formulation, subgrid scale models, bubbles and the origins of stabilized formulations, Comp. Meth. in Appl. Mech. and Eng.127, 387-401.

[32] Codina, R. (2000). Stabilization of incompressibility and convection through orthogonal sub-scales in finite element methods, Comp. Meth. in Appl. Mech. and Eng., 190, 1579-1599.

[33] Chiumenti, M., Valverde, Q., Agelet de Saracibar, C. and Cervera, M. (2002). A stabilized formulation for incompressible elasticity using linear displacement and pressure interpolations, Comp. Meth. in Appl. Mech. and Eng., 191, 5253-5264.

[34] Chiumenti, M., Valverde, Q., Agelet de Saracibar, C. and Cervera, M. (2003). A stabilized formulation for incompressible plasticity using linear triangles and tetrahedra, Int. J. of Plasticity, to appear.

[35] Cervera, M., Chiumenti, M., Valverde, Q. and Agelet de Saracibar, C. (2003). Mixed Linear/linear Simplicial Elements for Incompressible Elasticity and Plasticity. Comp. Meth. in Appl. Mech. and Eng., in press.

[36] Cervera, M., Chiumenti and Agelet de Saracibar, C. (2003). Softening, localization and stabilization: capture of discontinuous solutions in J2 plasticity. Submitted to Int. J. for Num. and Anal. Meth. in Geomechanics.

[37] Kachanov, L. M. (1958). Time of rupture process under creep conditions. Izvestia Akademii Nauk, Old Tech Nauk, 8, 26-31.

[38] Hughes, T.J.R., Feijoó, G.R., Mazzei. L., Quincy, J.B. (1998). The variational multiscale method-a paradigm for computational mechanics, Comp. Meth. in Appl. Mech. and Eng. 166, 3-28.

[39] Codina, R. (2002). Stabilized finite element approximation of transient incompressible flows using orthogonal subscales, Comp. Meth. in Appl. Mech. and Eng. 191, 4295-4321. 
[40] Baiocchi, C., Brezzi, F. and Franca, L. (1993). Virtual bubbles and Galerkin/least-squares type methods (Ga.L.S.). Comp. Meth. in Appl. Mech. and Eng. 105, 125-141.

[41] Brezzi, F., Bristeau, M.O., Franca, L., Mallet, M. and Rogé, G. (1992). A relationship between stabilized finite element methods and the Galerkin method with bubble functions. Comp. Meth. in Appl. Mech. and Eng. 96, 117-129.

[42] Hughes, T.J.R., Franca, L.P. and Balestra, M. (1986). A finite element formulation for computational fluid dynamics: V. Circumventing the BabuskaBrezzi condition: A stable Petrov-Galerkin formulation of the Stokes problem accomodating equal-order interpolations. Comp. Meth. in Appl. Mech. and Eng. 59, 85-99.

[43] Hughes, T.J.R., Franca, L.P. and Hulbert, G.M. (1989). A new finite element formulation for computational fluid dynamics: VIII. The Galerkin/leastsquare method for advective-diffusive equations. Comp. Meth. in Appl. Mech. and Eng. 73, 173-189.

[44] Cervera, M., Oliver, J., and Faria, R. (1995). Seismic evaluation of concrete dams via continuum damage models. Earth. Engng. Struc. Dyn., 24, 12251245 .

[45] Cervera, M., Oliver, J., and Manzoli, O. (1996). A rate-dependent isotropic damage model for the seismic evaluation of concrete dams. Earth. Engng. Struc. Dyn., 25, 987-1010.

[46] Finchant, S, La Borderie, C. and Pijaudier-Cabot, G. (1999). Isotropic and anisotropic descriptions of damage in concrete structures. Mechanics of Cohesive-Frictional Materials, 4, 339-359.

[47] Brezzi, F. and Fortin, M., 1991. Mixed and Hybrid Finite Element Methods, Spinger, New York.

[48] Hughes, T.J.R. (1995). Multiscale phenomena: Green's function, Dirichletto Neumann formulation, subgrid scale models, bubbles and the origins of stabilized formulations, Comp. Meth. in Appl. Mech. and Eng., 127, 387-401. 
[49] Codina, R. and Blasco, J. (1997). A finite element method for the Stokes problem allowing equal velocity-pressure interpolations, Comp. Meth. in Appl. Mech. and Eng. 143, 373-391.

[50] Codina, R. and Blasco, J. (2000). Stabilized finite element method for transient Navier-Stokes equations based on pressure gradient projection, Comp. Meth. in Appl. Mech. and Eng. 182, 287-300.

[51] Lemaitre, J. and Chaboche, J. L. (1978). Aspects phénoménologiques de la rupture par endommagement (in french). J. Méc. Appl., 2, 317-365.

[52] Simó, J. C. and Ju, J. W. (1987). Strain- and stress-based continuum damage models - I. formulation. Int. J. Solids and Structures, 23, 821-840.

[53] Ju, J. W. (1989). On energy-based coupled elastoplastic damage theories: Constitutive modeling and computational aspects. Int. J. Solids and Structures, 7, 803-833.

[54] Oliver, J. (2000). On the discrete constitutive models induced by strong discontinuity kinematics and continuum constitutive equations. Int. J. Solids and Structures, 37, 7207-7229.

[55] Bazant, Z.P. and Oh, B.H. (1983). Crack band theory for fracture of concrete. Material and Structures, 16, 155-177.

[56] Bazant, Z. and Pijaudier-Cabot, G., 1989. Measurement of characteristic length of non-local continuum. ASCE Journal of Engineering Mechanics, $115,755-767$.

[57] Jansen, D.C. and Shah, S.P. (1997). Effects of length on compressive strain softening of concrete. ASCE Journal of Engineering Mechanics, 123, 25-35.

[58] Oliver, J. (1989). A consistent characteristic length for smeared cracking models. Int. J. Num. Meth. Engng., 28, 461-474.

[59] Bicanic, N. and Pankaj (1990). Some computational aspects of tensile strain localization modelling in concrete. Eng. Frac. Mech., 35, 697-708. 
[60] Cervera, M., Agelet de Saracibar, C. and Chiumenti, M. (2002). COMET: COupled MEchanical and Thermal analysis. Data Input Manual, Version 5.0, Technical report IT-308, www.cimne.upc.es.

[61] GiD: the personal pre and post-processor (2002). www.gid.cimne.upc.es. 